\title{
Magnetohydrodynamic calculation of the temperature and wind velocity profile of the solar transition region. Preliminary results.
}

\author{
Todor M. Mishonov ${ }^{1, \star}$, Albert M. Varonov ${ }^{1}$, Nedeltcho I. Zahariev ${ }^{1}$, \\ Rositsa V. Topchiyska ${ }^{1}$, Boian V. Lazov ${ }^{1}$, and Stefan B. Mladenov ${ }^{1}$ \\ ${ }^{1}$ St. Clement of Ohrid University at Sofia, 5 J. Bourchier blvd., BG-1164 Sofia, Bulgaria
}

\begin{abstract}
The sharp almost step like increase the temperature in the transition region (TR) between chromosphere and solar corona is well-known from decades; for first time we are giving a detailed magnetohydrodynamic (MHD) calculation of the height dependence of the temperature. The width of the transition region is evaluated by maximal value of the logarithmic derivative of the temperature. At fixed heating, only MHD can give such a narrow width and in such sense, even the qualitative agreement with the observational data, gives the final verdict what the heating mechanism of the solar corona is. Static profiles of the temperature and wind velocity are calculated for static frequency dependent spectral density of the incoming MHD waves; no time dependent computer simulations. At fixed spectral density of MHD waves, the MHD calculation predicts height dependence of the non-thermal broadening of spectral lines and its angular dependence. For illustration is used one dimensional approximation of completely ionized hydrogen plasma in weak magnetic field, but it is considered that the width of the TR is weakly dependent with respect of further elaboration. The analyzed MHD calculation is a numerical confirmation of the qualitative concept of self-induced opacity of the plasma with respect to MHD waves. The plasma viscosity strongly increases with the temperature. Heated by MHD waves, plasma increases the wave absorption and this positive feedback leads to further heating. The static temperature profile is a result of a self-consistent calculation of propagation of MHD wave through the static background of wind and temperature profile. The numerical method allows consideration of incoming MHD waves with an arbitrary spectral density. Further elaboration of the method are briefly discussed: influence of second viscosity in the chromospheric part of the TR, influence of the magnetic field on the coronal side of the TR and investigation of such type effects on the width of the TR.
\end{abstract}

\section{Alfvén model for corona heating}

The discovery of the $5303 \AA$ green ${ }^{1}$ of $\mathrm{Fe}^{13+}$ and $6374 \AA$ red $^{2}$ of $\mathrm{Fe}^{9+}$ magneto-dipole transition lines in the solar corona spectrum [1] posed an important problem for the fundamental physics - what is the mechanism of the heating of the solar corona and why the temperature of the corona is 100 times

\footnotetext{
^e-mail: mishonov@gmail.com

$1 \quad$ Fe XIV, $\quad{ }^{2} \mathrm{P}_{3 / 2} \rightarrow{ }^{2} \mathrm{P}_{1 / 2}, \quad 3 \mathrm{~s}^{2} 3 \mathrm{p}^{1}$

$2 \mathrm{FeX}, \quad{ }^{2} \mathrm{P}_{1 / 2} \rightarrow{ }^{2} \mathrm{P}_{3 / 2}, \quad 3 \mathrm{~s}^{2} 3 \mathrm{p}^{5}$
} 
larger than the temperature of the photosphere. Those spectroscopic experiments put perhaps the most living unresolved problem in physics. None of the heating mechanisms has been confirmed and none has received an indisputable refutation. The 75 years old problem is still fresh and green.

The first idea by Alfvén [2] was that now called Alfvén waves (AW) [3] are the mechanism for heating the corona. Perhaps AW are generated by the turbulence in the convection zone and propagate along the magnetic field lines as through wave guides. Absorption is proportional to $\omega^{2}$ and the heating comes from high-frequency AW which are absorbed and not observed in the corona. Alfvén's idea for the viscous heating of plasma by absorption of AW was analyzed in the theoretical work by Heyvaerts [4]. In support of this idea is the work by Chitta [5] (Figures 8, 9 therein). The authors came to the conclusion that the spectral density of AW satisfies a power law with an index of 1.59. This gives a strong hint that this scaling can be extrapolated in the nearest spectral range for times less than $1 \mathrm{~s}$ and frequencies in the $\mathrm{Hz}$ range. Furthermore in the work by Tomczyk [6] it is stated that there exist very few direct measurements of the strength and orientation of coronal magnetic fields, meaning that the mechanisms responsible for heating the corona, driving the solar wind, and initiating coronal mass ejections remain poorly understood. After the launch of Hinode, Alfvènic type MHD modes were observed [7] and the well-forgotten spatially and temporally ubiquitous waves in the solar corona [8] came again into the limelight and gave strong support for the idea of Alfvén and the frequency of the notion AW as a key worth significantly increased.

A clear presence of outward and inward propagating waves in the corona was noted. $k-\omega$ diagnostics revealed coronal wave power spectrum with an exponent of $\approx-\frac{3}{2}$ (cf. Fig. 2 of [6]). The low frequency AW, on the other hand, reach the Earth orbit and thanks to the magnetometers on the various satellites we "hear" the basses of the great symphony of solar turbulence.

The observational data for the temperature profile of the solar corona show that the TR is extremely thin compared to the radius of the Sun [9-13]. All those observational data for the quiet-Sun chromosphere and transition region reveal one dimensional and time independent profiles which theoretically have to be derived by time averaged dynamic consideration. The width of the TR $\lambda$ might be evaluated by the maximum of logarithmic derivative of the temperature, $\lambda=\max \left(\frac{\mathrm{d} T}{T \mathrm{~d} x}\right)$. In order to qualitatively explain this small width, in [14] the idea of self-induced opacity of the plasma for Alfvén waves was introduced. A similar idea was analysed also by Suzuki [15] (see also [16-21]), however none of these articles is helpful for comparison with our manuscript because the width of the TR is not calculated in details in any of them. In the current paper we give a numerical realization of this idea by calculating for the first time the width of the TR using the framework of MHD.

First detailed observations by Skylab half a century ago revealed surprisingly that TR is very thin $\lambda$-equal in width to metropolitan Los Angeles [10]. As $\lambda$ is much smaller that the solar radius we have actually a plane one dimensional (1D) problem. In 1D the constant fluxes of mass, energy and momentum give 3 integrals of motion which significantly alleviate the calculations. We can eliminate the density $\rho(x)$ as dynamic variable and for the static profiles of temperature $T(x)$ and solar wind $U(x)$ we have to solve numerically first order differential equations.

Without a doubt the kinetic approach is indispensable for the treatment of low density solar corona but this problem is beyond the purpose of the present work. In our work we take into account the influence of the magnetic field on the viscosity within the hydrodynamic approach.

The purpose of the present work is to examine whether the initial Alfvén idea is correct and to solve the MHD equations which give the dependence of the temperature on the height $T(x)$ and the related velocity of the solar wind $U(x)$ supposing static spectral density of the incoming AW. We illustrate Alfvén's idea by an MHD calculation for completely ionized hydrogen plasma in the beginning of TR where the density of neutral Hydrogen atoms is already negligible. Due to the high density of the TR, MHD is an adequate tool to analyze the beginning of the process. 
Our starting point are the MHD equations [22] for the velocity field $\mathbf{v}$ and magnetic field $\mathbf{B}$

$$
\begin{aligned}
& \partial_{t} \rho+\operatorname{div} \mathbf{j}=0, \quad \mathbf{j}=\rho \mathbf{v}, \\
& \partial_{t}\left(\frac{1}{2} \rho \mathbf{v}^{2}+\varepsilon+\frac{\mathbf{B}^{2}}{2 \mu_{0}}\right)+\operatorname{div} \mathbf{q}=0, \\
& \partial_{t}(\rho \mathbf{v})+\nabla \cdot \boldsymbol{\Pi}=0,
\end{aligned}
$$

where

$$
\mathbf{q}=\rho\left(\frac{1}{2} \mathbf{v}^{2}+h\right) \mathbf{v}+\mathbf{v} \cdot \boldsymbol{\Pi}^{(\mathrm{visc})}-\varkappa \nabla T+\mathbf{S}
$$

is the energy density flux, $\rho$ is the mass density, $\varepsilon$ is the internal energy density, $x$ is the thermal conductivity, $h$ is the enthalpy per unit mass;

$$
\mathbf{S}=\frac{1}{\mu_{0}}\left[\mathbf{B} \times(\mathbf{v} \times \mathbf{B})-v_{\mathrm{m}} \mathbf{B} \times \operatorname{rot} \mathbf{B}\right],
$$

is the Poynting vector and $v_{\mathrm{m}} \equiv c^{2} \varepsilon_{0} \varrho$ is the magnetic diffusivity determined by Ohmic resistance $\varrho$ and vacuum susceptibility $\varepsilon_{0}$; vacuum permeability is $\mu_{0}$. For hot enough plasma $\varrho$ is negligible. The total momentum flux

$$
\boldsymbol{\Pi}=\rho \mathbf{v v}+P \mathbb{l}+\mathbf{\Pi}^{(\mathrm{visc})}+\boldsymbol{\Pi}^{(\mathrm{Maxw})}
$$

is a sum of the inviscid part $\rho \mathbf{v v}+P$ of the fluid, with pressure $P$ and

$$
\Pi_{i k}^{(\mathrm{visc})}=-\eta\left(\partial_{i} v_{k}+\partial_{k} v_{i}-\frac{2}{3} \delta_{i k} \nabla \cdot \mathbf{v}\right)-\zeta \delta_{i k} \nabla \cdot \mathbf{v},
$$

the viscous part of the stress tensor, with viscosity $\eta$ and second viscosity $\zeta$, and lastly, the Maxwell stress tensor

$$
-\Pi_{i k}^{(\text {Maxw })}=\frac{1}{\mu_{0}}\left(B_{i} B_{k}-\frac{1}{2} \mathbf{B}^{2} \delta_{i k}\right)
$$

with $\delta_{i k}$ the Kronecker delta. For temperatures above $10 \mathrm{kK}$ we treat coronal plasma as completely ionized hydrogen plasma with the following temperature dependence of kinetic coefficients [23]:

$$
\begin{gathered}
\varkappa=0.9 \frac{T^{5 / 2}}{e^{4} m^{1 / 2} \Lambda}, \quad \eta=0.4 \frac{M^{1 / 2} T^{5 / 2}}{e^{4} \Lambda}, \quad \zeta \approx 0, \\
\Lambda=\ln \left(\frac{r_{\mathrm{D}} T}{e^{2}}\right), \quad \frac{1}{r_{\mathrm{D}}^{2}}=\frac{4 \pi e^{2} n_{\mathrm{tot}}}{T}, \quad e^{2} \equiv \frac{q_{e}^{2}}{4 \pi \varepsilon_{0}}
\end{gathered}
$$

where $q_{e}$ is the electron charge, $m$ is the mass of electron, $M$ is the proton mass, $T$ is the temperature and $n_{\text {tot }}=n_{e}+n_{p}$ is the total density of electrons and protons; $\rho=M n_{p}$. We suppose that $\mu_{0}=4 \pi$ and $\varepsilon_{0}=1 / 4 \pi$, but in the practical system all formulae are the same; as well as in Heaviside-Lorentz units where $\mu_{0}=1$ and $\varepsilon_{0}=1$. As we mentioned above

$$
v_{\mathrm{m}}=\frac{c^{2}}{4 \pi} \frac{e^{2} m^{1 / 2} \Lambda}{0.6 T^{3 / 2}} \ll v_{\mathrm{k}} \equiv \frac{\eta}{\rho}=\frac{0.4 T^{5 / 2}}{e^{4} M^{1 / 2} n_{p} \Lambda}
$$

i.e. the hot hydrogen plasma is sticky, dilute, and "superconducting". Here $v_{\mathrm{k}}$ is the kinematic viscosity. We can introduce the magnetic Prandtl number as the ratio between the kinematic and the magnetic viscosity,

$$
\operatorname{Pr}_{\mathrm{m}}=\frac{\nu_{\mathrm{k}}}{\nu_{\mathrm{m}}}=\frac{0.96 \pi}{e^{6} c^{2} \sqrt{M m} n_{p} \Lambda^{2}(T)} T^{4}
$$


The coronal heating mechanism can be revealed without additional accessories. Let us mention also the relations $\varkappa \varrho=1.5 T / q_{e}^{2}$ leading to

$$
\varrho=\frac{1}{4 \pi \varepsilon_{0}} \frac{e^{2} m^{1 / 2} \Lambda}{0.6 T^{3 / 2}}
$$

and $\eta / \varkappa \approx \frac{4}{9} \sqrt{m M}$.

The applicability of our equations is governed by an additional condition:

$$
v_{p p}=\frac{1}{\tau_{p p}}=\frac{e^{2} n \Lambda}{T^{3 / 2} \sqrt{M}} \gg \omega_{B e}=\frac{q_{e} B}{M} .
$$

If in the dense plasma the frequency of the proton-proton collisions $v_{p p}$ is much smaller than the proton cyclotron frequency $\omega_{c}$, the magnetic field can be considered a perturbation and for the kinetic coefficients we can use the zero magnetic field formulas. An elementary gas kinetic consideration of main notions of plasma as proton-proton collision frequency $v_{p p}$ mean free time $\tau_{p p}$, thermal velocity $v_{T p}=\sqrt{T / M}$ mean free path $l_{p}=v_{T p} \tau_{p p}$ and other is given in the the appendices.

Lastly, we need to mention the existence of radiative losses. However, the thin TR has width much smaller not only than Earth's orbit radius but than Earth's radius and thus these losses are neglectable when considering the mechanism for coronal heating; continuum radiation may be neglected for all temperatures fewer than a few million degrees [24]. The hot corona exists in broad ranges where the heating mechanism cannot be effective. For this reason, in the narrow range of the transition region the radiation losses are negligible compared to the intensive heating, no matter what the concrete mechanism is. Quantitatively, this means that radiation power per unit volume $P_{\text {rad }} \ll n T U / \lambda$, where $n$ and $T$ are the number density and temperature of the corona, $\lambda$ is the width of the transition region, and $U$ is the velocity of the solar wind. The influence of gravitational field with acceleration $g_{\odot}$ is also negligible for the temperature gradient in the TR $M g_{\odot} \lambda \ll T$; cf. Ref. [20]

\section{MHD equations and energy fluxes}

First of all, we have to emphasize than MHD is not a model but adequate theory applicable for the dense plasma of TR. Let us now recall the main MHD equations.

The time derivative $\partial_{t} \mathbf{B}$ which implicitly participates in the energy conservation Eq. (2) obeys the equation cf. Ref. [22]

$$
\begin{aligned}
\mathrm{d}_{t} \mathbf{B} & =\mathbf{B} \cdot \nabla \mathbf{v}-\mathbf{B} \operatorname{div} \mathbf{v}-v_{\mathrm{m}} \Delta \mathbf{B}+\operatorname{grad} v_{\mathrm{m}} \times \operatorname{rot} \mathbf{B}, \\
\mathrm{d}_{t} & \equiv \partial_{t}+\mathbf{v} \cdot \nabla
\end{aligned}
$$

Analogously the momentum equation Eq. (3) can be rewritten by the substantial derivative

$$
\rho \mathrm{d}_{t} v_{i}=-\partial_{i} P+\partial_{k}\left\{\eta\left(\partial_{k} v_{i}+\partial_{i} v_{k}-\frac{2}{3} \delta_{i k} \partial_{j} v_{j}\right)\right\}+\partial_{i}\left(\zeta \partial_{j} v_{j}\right)-\frac{1}{\mu_{0}}(\mathbf{B} \times \operatorname{rot} \mathbf{B})_{i}
$$

We analyze AW propagating along magnetic field lines $\mathbf{B}_{0}$. We focus our attention on the narrow TR, where the static magnetic field is almost homogeneous and the waves are within acceptable accuracy one dimensional. For the velocity and magnetic fields we assume

$$
\begin{aligned}
\mathbf{v}(t, x) & =U(x) \mathbf{e}_{x}+u(t, x) \mathbf{e}_{z}, \\
\mathbf{B}(t, x) & =B_{0} \mathbf{e}_{x}+B_{0} b(t, x) \mathbf{e}_{z},
\end{aligned}
$$


with vertical homogeneous magnetic field $B_{0} \mathbf{e}_{x}$ perpendicular to the surface of the Sun. The transverse wave amplitudes of the velocity $u(t, x)$ and magnetic field $b(t, x)$ we represent with the Fourier integrals

$$
\begin{aligned}
& u(t, x)=\int_{-\infty}^{\infty} \tilde{u}(\omega, x) \mathrm{e}^{-\mathrm{i} \omega t} \frac{\mathrm{d} \omega}{2 \pi}, \\
& b(t, x)=\int_{-\infty}^{\infty} \tilde{b}(\omega, x) \mathrm{e}^{-\mathrm{i} \omega t} \frac{\mathrm{d} \omega}{2 \pi} .
\end{aligned}
$$

For illustrative purposes it is convenient to consider monochromatic AW with $u(t, x)=\hat{u}(x) \mathrm{e}^{-\mathrm{i} \omega t}$ and $b(t, x)=B_{0} \hat{b}(x) \mathrm{e}^{-\mathrm{i} \omega t}$. And later on to restore the summation on different frequencies which finally gives Fourier integration.

\subsection{Wave equations}

For transversal waves the general MHD equations Eq. (15) and Eq. (14) give the following exact system of linear for $\hat{u}(x)$ and $\hat{b}(x)$

$$
\begin{aligned}
\left(-\mathrm{i} \omega+U \mathrm{~d}_{x}\right) \hat{u} & =V_{\mathrm{A}}^{2} \mathrm{~d}_{x} \hat{b}+\frac{1}{\rho} \mathrm{d}_{x}\left(\eta \mathrm{d}_{x} \hat{u}\right)-v_{\mathrm{k}} k_{y}^{2} \hat{u}, \\
-\mathrm{i} \omega \hat{b} & =\mathrm{d}_{x} \hat{u}-\mathrm{d}_{x}(U \hat{b})+\mathrm{d}_{x}\left(v_{\mathrm{m}} \mathrm{d}_{x} \hat{b}\right)-v_{\mathrm{m}} k_{y}^{2} \hat{b},
\end{aligned}
$$

where

$$
V_{\mathrm{A}}(x)=B_{0} / \sqrt{\mu_{0} \rho(x)}
$$

is the Alfvén velocity. For illustrative purpose and complete lack of observational data in the present work we analyze only the case of zero transversal to the magnetic field wave vector $k_{y}=0$. This system can be Hamiltonized. In our numerical analysis we solve the first order linear system of equations

$$
-\mathrm{id}_{x} \Psi=\mathrm{K} \Psi, \quad \Psi \equiv\left(\begin{array}{c}
\hat{b} \\
\hat{u} \\
\hat{\gamma} \\
\hat{w}
\end{array}\right), \quad \Psi^{\dagger} \equiv\left(\hat{b}^{*}, \hat{u}^{*}, \hat{\gamma}^{*}, \hat{w}^{*}\right), \quad \mathrm{K} \equiv \frac{\mathrm{i} \overline{\mathrm{M}}}{v_{\mathrm{m}} \nu_{\mathrm{k}}},
$$

where $\hat{\gamma} \equiv \mathrm{d}_{x} \hat{b}, \hat{w} \equiv \mathrm{d}_{x} \hat{u}$ and

$$
\overline{\mathrm{M}} \equiv\left(\begin{array}{cccc}
0 & 0 & -v_{\mathrm{m}} \nu_{\mathrm{k}} & 0 \\
0 & 0 & 0 & -v_{\mathrm{m}} v_{\mathrm{k}} \\
\left(\mathrm{i} \omega-\mathrm{d}_{x} U\right) \nu_{\mathrm{k}} & 0 & -\left(U+\mathrm{d}_{x} v_{\mathrm{m}}\right) v_{\mathrm{k}} & v_{\mathrm{k}} \\
0 & \mathrm{i} \omega v_{\mathrm{m}} & V_{\mathrm{A}}^{2} \nu_{\mathrm{m}} & -\left(U-\frac{\mathrm{d}_{x}(\eta)}{\rho}\right) \nu_{\mathrm{m}}
\end{array}\right)
$$

For homogeneous medium with constant $\eta, v_{\mathrm{m}}, \rho, V_{\mathrm{A}}$, and $U$, in short for constant wave-vector matrix $\mathrm{K}$, the exponential substitution $\Psi \propto \exp (\mathrm{i} k x)$ in Eq. (22) or equivalently Eq. (19) and Eq. (20) gives the secular equation $\operatorname{det}(\mathrm{K}-k 1 \mathrm{l})=0$, which after some algebra gives the dispersion equation of $\mathrm{AW}$

$$
\left(\omega_{\mathrm{D}}+\mathrm{i} v_{\mathrm{m}} k^{2}\right)\left(\omega_{\mathrm{D}}+\mathrm{i} v_{\mathrm{k}} k^{2}\right)=V_{\mathrm{A}}^{2} k^{2},
$$

where $\omega_{\mathrm{D}} \equiv \omega-k U$ is the Doppler shifted frequency. Usually either viscosity coefficient is significantly bigger than the other. For example, for hot plasma $v_{\mathrm{k}} \gg v_{\mathrm{m}}$ and with an acceptable approximation we can neglect the smaller one and to work with matrix $3 \times 3$. Approximation of ideal 
fluid is reduced to both negligible viscosity coefficients and corresponding $2 \times 2$ matrix gives the wellknown AW dispersion in moving fluid $\omega_{\mathrm{D}}^{2}=V_{\mathrm{A}}^{2} k^{2}$. A perturbative treatment of dispersion equation $\omega(k)=\omega^{\prime}+i \omega^{\prime \prime}$ Eq. (23) gives [22] $\omega^{\prime \prime}=-\frac{1}{2}\left(v_{\mathrm{m}}+v_{\mathrm{k}}\right) k^{2}$. If WKB approximation is applicable for wave amplitude damping we have to use the eigenvalue of $\mathrm{K}$ matrix giving smallest damping for right running wave.

In order to make model evaluation of the influence of magnetic field on viscosity for the waves we can use the the Braginskii result [25]

$$
v_{\mathrm{k}, 2}=\frac{\frac{6}{5} \tilde{x}^{2}+\frac{7}{3}}{\tilde{x}^{4}+4.03 \tilde{x}^{2}+\frac{7}{3}} v_{\mathrm{k}},
$$

where $\tilde{x} \equiv \omega_{B e} \tau_{p p}$.

\subsection{Wind variables}

We solve the wave equation Eq. (22) from some height at which hydrogen plasma is approximately completely ionized $x=0$ to some distance large enough $x=l$, where the short wavelength AW are almost absorbed. As we pointed out, this distance is much bigger than the width of the transition region $\lambda$, but much smaller than solar radius. That is why the problem is effectively one dimensional. The horizontal wavelength of the waves responsible for heating is much smaller than the range of area for which we are calculating the temperature profile.

The considered one-dimensional $0<x<l$ time independent problem has three integrals corresponding to the three conservation laws related to mass, energy and momentum. The mass conservation law Eq. (1) gives the constant flow

$$
j=\rho(x) U(x)=\rho_{0} U_{0}=\rho_{l} U_{l}=\text { const },
$$

where $\rho_{0}=\rho(0), \rho_{l}=\rho(l), U_{0}=U(0)$, and $U_{l}=U(l)$. The energy conservation law reduces to a constant flux along the $x$-axis

$$
\begin{aligned}
& q=q_{\text {wind }}^{\text {ideal }}(x)+\tilde{q}(x)=\text { const }, \\
& q_{\text {wind }}^{\text {ideal }}=q_{U}+q_{P}, \quad q_{U} \equiv\left(\frac{1}{2} U^{2}\right) \rho U, \quad q_{P} \equiv h \rho U .
\end{aligned}
$$

Here the first term describes the energy of the ideal wind, i.e. a wind from an ideal (inviscid) fluid. The second term $\tilde{q}(x)$ includes all other energy fluxes; in our notations tilde will denote sum of the non-ideal (dissipative) terms of the wind and wave terms. In detail the non-ideal part of the energy flux $\tilde{q}(x)$ consists of: the wave kinetic energy $\propto|\hat{u}|^{2}$, viscosity (wind $\propto \frac{4}{3} \eta+\zeta$ and wave $\propto \eta$ components), heat conductivity $\propto \varkappa$, and Poynting vector $\propto \hat{b}^{*}$,

$$
\begin{gathered}
\tilde{q}(x) \equiv q_{\xi}+q_{\varkappa}+q_{\text {wave }}, \\
q_{\xi} \equiv-\xi U \mathrm{~d}_{x} U, \quad q_{\varkappa} \equiv-\varkappa \mathrm{d}_{x} T, \quad q_{\text {wave }}=q_{\text {wave }}^{\text {ideal }}+q_{\text {wave }}^{\text {diss }}, \\
q_{\text {wave }}^{\text {ideal }}=q_{u}+q_{b}+q_{u b}, \quad q_{\text {wave }}^{\text {diss }}=q_{\eta}+q_{\varrho}, \\
q_{u} \equiv \frac{j}{4} \sum_{\text {waves }}|\hat{u}|^{2}, \quad q_{b} \equiv \frac{B_{0}^{2}}{2 \mu_{0}} \sum_{\text {waves }} U|\hat{b}|^{2}, \quad q_{u b} \equiv-\frac{B_{0}^{2}}{2 \mu_{0}} \sum_{\text {waves }} \operatorname{Re}\left(\hat{b}^{*} \hat{u}\right), \\
q_{\eta}=-\frac{1}{4} \eta \sum_{\text {waves }} \mathrm{d}_{x}|\hat{u}|^{2}, \quad q_{\varrho}=-\frac{B_{0}^{2}}{2 \mu_{0}} v_{\mathrm{m}} \sum_{\text {waves }} \operatorname{Re}\left(\hat{b}^{*} \hat{\gamma}\right),
\end{gathered}
$$




$$
\begin{aligned}
& q_{\text {wave }}^{\text {ideal }}=\sum_{\text {waves }}\left\{\frac{j}{4}|\hat{u}|^{2}+\frac{B_{0}^{2}}{2 \mu_{0}}\left[U|\hat{b}|^{2}-\operatorname{Re}\left(\hat{b}^{*} \hat{u}\right)\right]\right\}, \quad q_{\text {wave }}^{\text {diss }}=\sum_{\text {waves }}\left\{-\frac{1}{4} \eta \mathrm{d}_{x}|\hat{u}|^{2}-\frac{B_{0}^{2}}{2 \mu_{0}} v_{\mathrm{m}} \operatorname{Re}\left(\hat{b}^{*} \hat{\gamma}\right)\right\}, \\
& q_{\text {wave }}=\sum_{\text {waves }}\left\{\frac{j}{4}|\hat{u}|^{2}-\frac{1}{4} \eta \mathrm{d}_{x}|\hat{u}|^{2}+\frac{B_{0}^{2}}{2 \mu_{0}}\left(U|\hat{b}|^{2}-\operatorname{Re}\left(\hat{b}^{*} \hat{u}\right)-v_{\mathrm{m}} \operatorname{Re}\left(\hat{b}^{*} \hat{\gamma}\right)\right)\right\},
\end{aligned}
$$

where $\xi \equiv \frac{4}{3} \eta+\zeta$. In order to alleviate the detail analysis depicted in Fig. 3, we have introduced convenient notations for all terms with different nature. Here time averaged energy flux is represented by the amplitudes of the monochromatic oscillations, this is a standard procedure for alternating current processes. In our case we have, for example, $\left\langle\hat{u}^{2}\right\rangle_{t}=\left\langle(\operatorname{Re} \hat{u})^{2}\right\rangle_{t}=\left\langle\frac{1}{4}\left(\hat{u}+\hat{u}^{*}\right)^{2}\right\rangle_{t}=\frac{1}{2}|\hat{u}|^{2}$. The other terms from Eq. (4) are averaged in a similar way like in the equation above. In short, we substituted the formulas for AW Eq. (16) propagating along the magnetic field in $x$-direction in the general formula for the energy flux Eq. (4) and made the time averaging and summation over waves with different frequencies. We will repeat the same approach to the momentum flux Eq. (6)

For constant spectrum of incoming waves the momentum conservation law Eq. (6) gives constant flux $\Pi=\Pi_{x x}$

$$
\begin{aligned}
& \Pi=\Pi_{\text {wind }}^{\text {ideal }}(x)+\tilde{\Pi}(x)=\text { const }, \\
& \Pi_{\text {wind }}^{\text {ideal }}=\Pi_{U}+\Pi_{P}, \quad \Pi_{U} \equiv \rho U U, \quad \Pi_{P} \equiv P,
\end{aligned}
$$

the sum of the ideal wind fluid and the other terms

$$
\tilde{\Pi}(x) \equiv \Pi_{\xi}+\Pi_{\text {wave }}, \quad \Pi_{\xi} \equiv-\xi \mathrm{d}_{x} U, \quad \Pi_{\text {wave }} \equiv \sum_{\text {waves }} \frac{1}{4 \mu_{0}}|\hat{b}|^{2},
$$

which take into account the wave part of the Maxwell stress tensor $\propto|\hat{b}|^{2}$ and viscosity of the wind $\propto \xi$. The sum here and in Eq. (27) is actually integral over the spectral density $\mathcal{W}(\omega)$ of incoming AW

$$
\Pi_{\text {wave }} \equiv \sum_{\text {waves }} \frac{1}{4 \mu_{0}}|\hat{b}|^{2}=\int_{0}^{\infty} \frac{\mathrm{d} \omega}{2 \pi} \mathcal{W} \frac{1}{4 \mu_{0}}|\hat{b}|^{2} .
$$

Let us mention that all components of the wave $\Psi(\omega, x)$ have frequency dependence. One can suppose that the spectral density $\mathcal{W}$ of the waves coming from the chromosphere has the same power law dependence, i.e.

$$
\mathcal{W}_{\bar{\omega}}=C / \omega^{\alpha} .
$$

The parameter $\alpha$ is between 1.5 and 2: $\frac{3}{2}$ [6], 1.59 [5], 2 [26]. $C$ is the unknown parameter of the theory, which we vary for fixed $\xi$ in order to reproduce the temperature increase in the TR. In our earlier works [27] simulations of plasma heating with power law spectral density is given with up to 8 AW with different frequencies.

We have to solve the hydrodynamic problem for calculation of wind velocity and temperature at known energy and momentum fluxes. The problem is formally reduced to analogous one for a jet engine, cf. Ref. [28]. We approximate the corona as completely ionized hydrogen plasma, i.e. electrically neutral mixture of electrons and protons. The experimental data tells us that proton temperature $T_{p}$ is higher than electron one $T_{e}$. This is an important hint that heating goes through the viscosity determined mainly by protons. However for illustration purpose and simplicity we assume proton and electron temperatures to be equal $T_{e}=T_{p}=T$. For such an ideal (in thermodynamic sense) gas the 
local sound velocity is

$$
\begin{aligned}
& c_{\mathrm{s}}^{2}(x)=\frac{c_{p}}{c_{v}} \frac{P}{\rho}=\gamma \frac{T}{\langle m\rangle}, \quad \gamma=\frac{c_{p}}{c_{v}}=\frac{5}{3}, \quad\langle m\rangle=\frac{n_{p} M+n_{e} m}{n_{p}+n_{e}} \approx \frac{1}{2} M, \\
& n_{e}=n_{p}=\frac{1}{2} n_{\mathrm{tot}}, \quad P=n_{\mathrm{tot}} T=\frac{\rho T}{\langle m\rangle}=\frac{j}{U} \frac{T}{\langle m\rangle}, \quad h=c_{p} \frac{T}{\langle m\rangle}=\frac{\varepsilon+P}{\rho},
\end{aligned}
$$

where, as we mentioned earlier, $h$ is the enthalpy per unit mass and $\varepsilon$ is the density of internal energy. Although there are some hints for different values of the adiabatic index $\gamma$ [29], we will use the traditional value of $5 / 3$ for our calculations since this choice will not change the essence of our presentation. Hydrogen plasma is well theoretically investigated and the $5 / 3$ value of gamma is used in hundreds of works. There are no theoretical hints for significant deviations from this value obtained by ideal gas approximation. Actually a small variation of the value of gamma does not change qualitatively the temperature profile of the TR.

In order to alleviate the final formulae we introduce two dimensionless variables $\chi$ and $\tau$ which represent the non-ideal part of the energy and momentum flux respectively

$$
\left.\chi(x) \equiv \frac{\tilde{q}(x)}{\rho_{0} U_{0}^{3}}\right|_{x} ^{0},\left.\quad \tau(x) \equiv \frac{\tilde{\Pi}(x)}{\rho_{0} U_{0}^{2}}\right|_{x} ^{0} .
$$

Here we could include gravitational field, Bremsstrahlung or other accessories, which are negligible for the narrow TR. Analogously, for the wind velocity and temperature we have

$$
\bar{U}(x) \equiv \frac{U(x)}{U_{0}}, \quad \Theta(x) \equiv \frac{T(x)}{\langle m\rangle U_{0}^{2}}, \quad \Theta_{0}=\Theta(0),
$$

where $U_{0}=U(0)$. The energy and momentum constant fluxes Eq. (26) and Eq. (28) in the new notation take the form

$$
\begin{aligned}
& \frac{q-\tilde{q}(0)}{\rho_{0} U_{0}^{3}}=\frac{1}{2} \bar{U}^{2}+c_{p} \Theta-\chi=\frac{1}{2}+c_{p} \Theta_{0}, \\
& \frac{\Pi-\tilde{\Pi}(0)}{\rho_{0} U_{0}^{2}}=\bar{U}+\Theta / \bar{U}-\tau=1+\Theta_{0} .
\end{aligned}
$$

From the second equation we express the dimensionless temperature $\Theta$ and substitute in the first one. Solving the quadratic equation for the wind velocity $U$ we derive

$$
U=U_{0} \bar{U}, \quad \bar{U}(x)=\frac{1}{\gamma+1}\left(\gamma+s^{2}+\gamma \tau(x)-\sqrt{\mathcal{D}(x)}\right),
$$

where for the discriminant we have

$$
\begin{aligned}
& \mathcal{D}=\left(s^{2}-1\right)^{2}-2 \chi\left(\gamma^{2}-1\right)+\gamma \tau\left[\gamma \tau+2\left(\gamma+s^{2}\right)\right], \\
& s^{2} \equiv \frac{c_{\mathrm{s}}^{2}(0)}{U_{0}^{2}}=\gamma \Theta_{0}, \quad c_{\mathrm{s}}^{2}(x)=\left(\frac{\partial P}{\partial \rho}\right)_{S}=\frac{\gamma T(x)}{\langle m\rangle} .
\end{aligned}
$$

If $\chi=0$ and $\tau=0$, we get the initial condition $\left.\bar{U}\right|_{\chi=0, \tau=0}=1$. This condition determines the sign in front of $\sqrt{\mathcal{D}}$ in Eq. (36). Here $\gamma$ is the constant ratio of the heat capacities, and $s \equiv c_{\mathrm{S}}(0) / U_{0}$ is the ratio of the sound and wind velocity at $x=0$. We suppose that initial wind velocity is very 
small $U(0) \ll c_{\mathrm{s}}(0)$. The velocity distribution Eq. (36) can be substituted in Eq. (35) and we derive the dimensionless equation for the temperature distribution expressed by the wind velocity profile

$$
\begin{aligned}
& T(x)=\langle m\rangle U_{0}^{2} \Theta(x), \\
& \Theta(x)=\bar{U}(x)\left[1+\Theta_{0}+\tau(x)-\bar{U}(x)\right], \quad \Theta_{0}=\frac{s^{2}}{\gamma} .
\end{aligned}
$$

The solutions for velocity $\bar{U}(x)$ Eq. (36) and temperature $\bar{T}(x)$ Eq. (38) distributions are important ingredients in our analysis and derivation of the self-consistent picture of the solar wind. We use a one dimensional approximation and in addition the constant flux of mass, energy and momentum give 3 integrals of motion. This enables us to solve exactly the nonlinear part the MHD equations for the solar corona heating problem analytically. As the energy and momentum flux density equations, Eq. (26) and Eq. (28) respectively, contain space derivatives, these nonlinear equations are differential equations, in which the variables are explicitly expressed but not the derivatives, as it is usual.

The intuitive interpretation is unnecessary, but nevertheless rewriting Eq. (34) and Eq. (35) as

$$
\begin{aligned}
& \frac{1}{2} \bar{U}^{2}+c_{p} \Theta=\frac{1}{2}+c_{p} \Theta_{0}+\chi, \\
& \bar{U}+\Theta / \bar{U}=1+\Theta_{0}+\tau,
\end{aligned}
$$

one can say that $\chi(x)$ is the energy given from all non-ideal terms to the initial energy flux of the ideal wind $\frac{1}{2}+c_{p} \Theta_{0}$ in order ideal wind flux to reach his value $\frac{1}{2} \bar{U}^{2}+c_{p} \Theta$ at coordinate $x$. In the same dimensionless units $\rho_{0}=1$ and $U_{0}=1$ one can consider the solar wind as a wind of ideal fluid with initial momentum density $1+\Theta_{0}$ in $x=0$. In the space interval $(0, x)$ this ideal wind obtains from non-ideal processes momentum flux $\tau(x)$ and finally the momentum flux at coordinate $x$ is $\bar{U}+\Theta / \bar{U}$. Such a chain of notions could be useful to trace influence of every process to the wind velocity $U(x)$ and temperature $T(x)$ profiles.

\subsection{Boundary conditions for the waves}

At known background wind variables $U(x)$ and $T(x)$ we can solve the wave equation Eq. (22) for run-away AW at $x=l$. As we will see later the run-away boundary condition Eq. (55) corresponds to right propagating AW at the right boundary of the interval. The wave equation Eq. (22) is extremely stiff at small viscosity, and numerical solution is possible to be obtained only downstream from $x=0$ to $x=l$. We have to find the linear combination of left and right propagating waves at $x=0$, which gives the run-away condition at $x=l$.

The solution of wave equation according to Eq. (26) determines the energy flux related to the propagation of AW

$$
\tilde{q}_{\text {wave }}(\Psi(x)) \equiv \Psi^{\dagger} g \Psi=\frac{j}{4}|\hat{u}|^{2}-\frac{1}{2} \eta \operatorname{Re}\left(\hat{u}^{*} \hat{w}\right)-\frac{\nu_{\mathrm{m}} B_{0}^{2}}{2 \mu_{0}} \operatorname{Re}\left(\hat{b}^{*} \mathrm{~d}_{x} \hat{b}\right)+\frac{B_{0}^{2}}{2 \mu_{0}}\left(U|\hat{b}|^{2}-\operatorname{Re}\left(\hat{b}^{*} \hat{u}\right)\right),
$$

where

$$
g(x) \equiv\left(\begin{array}{cccc}
\frac{U B_{0}^{2}}{2 \mu_{0}} & -\frac{B_{0}^{2}}{4 \mu_{0}} & -\frac{\nu_{\mathrm{m}}}{4 \mu_{0}} B_{0}^{2} & 0 \\
-\frac{B_{0}^{2}}{4 \mu_{0}} & \frac{1}{4} j & 0 & -\frac{1}{4} \eta \\
-\frac{\nu_{\mathrm{m}}}{4 \mu_{0}} B_{0}^{2} & 0 & 0 & 0 \\
0 & -\frac{1}{4} \eta & 0 & 0
\end{array}\right) .
$$


Here $j$-term represents kinetic energy of the wave, $\eta$-term comes from the viscous part of the wave energy flux, and $B_{0}$-terms describe the Poynting vector of the wave.

For hot plasmas the Ohmic dissipation is negligible and we can use the approximation $v_{\mathrm{m}}=0$. In this case it is not necessary to use dynamic variable $\hat{\gamma}$ and we can work with $3 \times 3$ matrix. For zero viscosity and Ohmic ressisitivity, equations for AW amplitudes can be reduced to 1D Schrödinger equation [30]. For the illustration of the wave boundary values we will use this approach.

In order to take into account the boundary condition at $x=l$, we calculate the eigenvectors of the matrix K, which according to Eq. (22) determine the wave propagation in a homogeneous fluid with amplitude $\propto \exp (\mathrm{i} k x)$. Then the eigenvalues of $\mathrm{K}$ give the complex wave-vectors

$$
k=k^{\prime}+\mathrm{i} k^{\prime \prime}=\text { eigenvalue }(\mathrm{K}), \quad \text { i.e. } \quad \operatorname{det}(\mathrm{K}-k 1)=0 .
$$

The three eigenvectors L, D and R are ordered by spatial decrements of their eigenvalues

$$
k_{\mathrm{L}}^{\prime \prime}<0<k_{\mathrm{R}}^{\prime \prime}<k_{\mathrm{D}}^{\prime \prime}
$$

and are normalized by the conditions

$$
-\mathrm{L}^{\dagger} g \mathrm{~L}=\mathrm{R}^{\dagger} g \mathrm{R}=\mathrm{D}^{\dagger} g \mathrm{D}=1
$$

where the sign corresponds to the direction of wave propagation. Notation L corresponds to left propagating wave, $\mathrm{R}$ to right propagating wave, and $\mathrm{D}$ for an overdamped at small viscosity mode.

For technical purposes we introduce the matrix notations

$$
\mathrm{L}=\left(\begin{array}{c}
\mathrm{L}_{u}(x) \\
\mathrm{L}_{b}(x) \\
\mathrm{L}_{w}(x)
\end{array}\right), \quad \mathrm{R}=\left(\begin{array}{c}
\mathrm{R}_{u}(x) \\
\mathrm{R}_{b}(x) \\
\mathrm{R}_{w}(x)
\end{array}\right), \quad \mathrm{D}=\left(\begin{array}{c}
\mathrm{D}_{u}(x) \\
\mathrm{D}_{b}(x) \\
\mathrm{D}_{w}(x)
\end{array}\right)
$$

For low enough frequencies $\omega \rightarrow 0$ and wind velocities the modes describe: 1) right-propagating AW with $k_{\mathrm{R}}^{\prime} \approx \omega / V_{\mathrm{A}}$ and small $\left.k_{\mathrm{R}}^{\prime \prime} \approx v_{\mathrm{k}} \omega^{2} / 2 V_{\mathrm{A}}^{3} \ll k_{\mathrm{R}}^{\prime}, 2\right)$ left propagating wave $k_{\mathrm{L}}=-k_{\mathrm{R}}$, and a diffusion overdamped mode $k_{\mathrm{D}}^{\prime \prime} \approx V_{\mathrm{A}}^{2} / \nu_{\mathrm{k}} U \gg k_{\mathrm{D}}^{\prime}$ which describes the drag of a static perturbation by the slow wind $U \ll V_{\mathrm{A}}$ in a fluid with small viscosity. In this low frequency and long wavelength limit the stiffness ratio of the eigenvalues is very large

$$
r_{\mathrm{DR}}=\frac{\left|k_{\mathrm{D}}\right|}{\left|k_{\mathrm{R}}\right|} \approx \frac{k_{\mathrm{D}}^{\prime \prime}}{k_{\mathrm{R}}^{\prime}} \approx \frac{V_{\mathrm{A}}^{3}}{v_{\mathrm{k}} U \omega} \gg 1 .
$$

As we emphasized the wave equations Eq. (22) form a very stiff system and indispensably has to be solved downstream from the chromosphere $x=0$ to the corona $x=l$ using algorithms for stiff systems. Let

$$
\psi_{\mathrm{L}}(x)=\left(\begin{array}{c}
u_{\mathrm{L}}(x) \\
b_{\mathrm{L}}(x) \\
w_{\mathrm{L}}(x)
\end{array}\right), \quad \psi_{\mathrm{R}}(x)=\left(\begin{array}{c}
u_{\mathrm{R}}(x) \\
b_{\mathrm{R}}(x) \\
w_{\mathrm{R}}(x)
\end{array}\right)
$$

are the solutions of the wave equation Eq. (22) with boundary conditions

$$
\psi_{\mathrm{L}}(0)=\mathrm{L}(0), \quad \psi_{\mathrm{R}}(0)=\mathrm{R}(0) .
$$

We look for a solution as a linear combination

$$
\psi(x)=\psi_{\mathrm{R}}(x)+r \psi_{\mathrm{L}}(x),
$$


in other words we suppose that from the low viscosity chromosphere plasma do not come overdamped diffusion modes. The strong decay rate make them negligible at $x=0$. Physically this means that AW (R-modes) are coming from the Sun and some of them are reflected from the TR (L-modes)

$$
\psi(0)=\mathrm{R}(0)+r \mathrm{~L}(0)
$$

Analogously for the configuration of open corona we have to take into account the run-away boundary condition for which we suppose zero amplitude for the wave coming from infinity

$$
\psi(l)=\tilde{t} \mathrm{R}(l)+\tilde{c} \mathrm{D}(l) .
$$

Written by components

$$
\left(\begin{array}{c}
u_{\mathrm{R}}(l) \\
b_{\mathrm{R}}(l) \\
w_{\mathrm{R}}(l)
\end{array}\right)+r\left(\begin{array}{c}
u_{\mathrm{L}}(l) \\
b_{\mathrm{L}}(l) \\
w_{\mathrm{L}}(l)
\end{array}\right)=\tilde{t}\left(\begin{array}{c}
\mathrm{R}_{u}(l) \\
\mathrm{R}_{b}(l) \\
\mathrm{R}_{w}(l)
\end{array}\right)+\tilde{c}\left(\begin{array}{c}
\mathrm{D}_{u}(l) \\
\mathrm{D}_{b}(l) \\
\mathrm{D}_{w}(l)
\end{array}\right) .
$$

This boundary condition gives a linear system of equation for the reflection coefficient $r$, transmission coefficient $\tilde{t}$ and the mode-conversion coefficient $\tilde{c}$.

For $l \rightarrow \infty$ when $\exp \left[-k_{\mathrm{D}}^{\prime \prime}(l) l\right] \ll 1 \exp \left[-k_{\mathrm{R}}^{\prime \prime}(l) l\right]$ the amplitude of D-mode is negligible and the run-away boundary condition reads

$$
\psi(l)=\psi_{\mathrm{R}}(l)+r \psi_{\mathrm{L}}(l) \approx \tilde{t} \mathrm{R}(l),
$$

or by components

$$
\left(\begin{array}{c}
u_{\mathrm{R}}(l) \\
b_{\mathrm{R}}(l)
\end{array}\right)+r\left(\begin{array}{c}
u_{\mathrm{L}}(l) \\
b_{\mathrm{L}}(l)
\end{array}\right)=\tilde{t}\left(\begin{array}{l}
\mathrm{R}_{u}(l) \\
\mathrm{R}_{b}(l)
\end{array}\right) .
$$

These systems give the amplitudes of the reflected wave $r$ and transmitted wave $\tilde{t}$ in the solution Eq. (51). For this solution we have the energy flux of transmitted $\mathcal{T}$ and reflected $\mathcal{R}$ waves

$$
\begin{aligned}
& \mathcal{T} \equiv \psi^{\dagger}(l) g(l) \psi(l)=|\tilde{t}|^{2}+|\tilde{c}|^{2}+\left(\tilde{t} \tilde{c}^{*} \mathrm{D}^{\dagger}(l) g(l) \mathrm{R}(l)+\text { c.c. }\right), \\
& 1-\mathcal{R} \equiv \psi^{\dagger}(0) g(0) \psi(0)=1-|r|^{2}+\left(r^{*} \mathrm{~L}^{\dagger}(0) g(0) \mathrm{R}(0)+\text { c.c. }\right) .
\end{aligned}
$$

Then we introduce the absorption coefficient

$$
\mathcal{A} \equiv-\left.\psi^{\dagger}(x) g(x) \psi(x)\right|_{0} ^{l}=1-\mathcal{R}-\mathcal{T} .
$$

The described solution is normalized by unit energy flux of the R-wave. If we wish to fix energy flux of the right propagating wave to be $q_{\text {wave }}(0)$ we have to make the renormalization

$$
\Psi(x)=A_{\text {wave }} \psi(x),
$$

using a parameter $A_{\text {wave. }}$. In this section we have described Absorbing Boundary Conditions $(\mathrm{ABC})[31]$ well known from radar calculations, but realization for AW is more complicated and require eigenvector analysis. Now using $\Psi(x)$ we can calculate the wave part of the energy flux Eq. (42) and the wave part of the momentum flux

$$
\tilde{\Pi}_{\text {wave }}(x) \equiv \frac{1}{4 \mu_{0}}|\hat{b}(x)|^{2} .
$$

If we work with $4 \times 4$ matrix the generalization is obvious, in this case we have to operate with one additional diffusion mode. 


\section{Self-consistent procedure and results}

We have to say few words about the numerical methods. For high frequency waves we can use WKB approximation, which for our problem reads

$$
\Psi(\omega, x)=\sqrt{\mathcal{W}(\omega)} \mathrm{R}(\mathrm{x}) \exp \left(\mathrm{i} \int_{0}^{x} k_{\mathrm{R}}\left(x^{\prime}\right) \mathrm{d} x^{\prime}\right) .
$$

As the equations for wind variables are stiff, we can use the implicit Euler method for $T(x)$ and $U(x)$

$$
\frac{\mathrm{d} y}{\mathrm{~d} x}=f(x, y), \quad y(x+h)=y(x)+f(x+h, y(x+h)) .
$$

For the realization of this method we use the explicit formulas Eq. (36) and Eq. (38) for $U$ and $T$ respectively. As dissipative fluxes contains the derivatives $-\xi U \mathrm{~d}_{x} U-\varkappa \mathrm{d}_{x} T$ for the energy flux Eq. (27) and $\tilde{\Pi}_{\xi}(x) \equiv-\xi \mathrm{d}_{x} U$ in (29), the explicit expression for the wind variables Eq. (36) and Eq. (38) is actually a system of first order ordinary differential equations. In the WKB approximation complete system for wind and wave variables can be solved simultaneously.

One alternative is to use explicit expressions for derivatives $\mathrm{d}_{x} U$ and $\mathrm{d}_{x} T$ which age given from the constant energy Eq. (26) and momentum Eq. (28) fluxes. The limit case of ideal fluid with small $\xi$ and $x$ in the denominators gives the singular perturbation theory. Care is therefore needed for the choice of the numerical method.

For exact wave variables the implicit Euler method applied for Eq. (22) gives

$$
\Psi(x+h)=[1-\mathrm{i} h \mathrm{~K}(x+h)]^{-1} \Psi(x) .
$$

For this case the WKB approximation is only a starting point. Then for fixed wind profiles $T$ and $U$ we solve the wave equation with the corresponding boundary conditions. And using wave fluxes of energy and momentum we calculate the next approximation for the wind variables $T$ and $U$. The procedure has to be repeated to reach self-consistency of wind and wave variables.

The simple Euler method can be elaborated by incorporation of predictor-corrector method using for small interpolation the Eitken formula for polynomial interpolation. Sequential polynomial interpolations can be rearranged as Padé interpolation using well-known epsilon algorithm by Wynn and Backer; some details are given in the Appendix II.

Following the described numerical method we have calculated the temperature and wind velocity profiles and the results are depicted in Fig. 1 and Fig. 2.

The energy and momentum flux density of the calculated temperature and wind profiles are respectively given in Fig. 3 and Fig. 4.

Finally, we present a comparison between theory and observation in Fig. 5: the calculated temperature profile from Fig. 1 is placed on the presented observational data from Ref. [13].

\section{Conclusion}

\subsection{Discussions}

In spite that iron was suspicious from the very beginning the problem of Coronium was a 70 year standing mystery until unambiguous identification as $\mathrm{Fe}^{13+}$ by Grotrian and Edlen [33] in 1937. The same 70 year time quantum was repeated. In 1947 Alfvén [2] advocated the idea that absorption of AW is the mechanism of heating of solar corona. Unfortunately the idea by Swedish iconoclast [34] was never realized in its original form: what can be calculated, what is measured, what is explained 


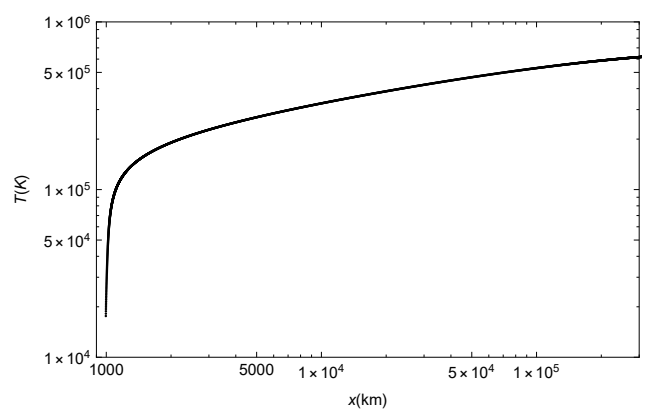

Figure 1: Temperature profile. Height dependence of the temperature $\mathrm{T}(\mathrm{x})$. MHD calculations qualitatively reproduce the well-known observational data $[12,13,32]$. This step-like behaviour confirms the scenario of self-induced opacity with respect to Alfvén waves.

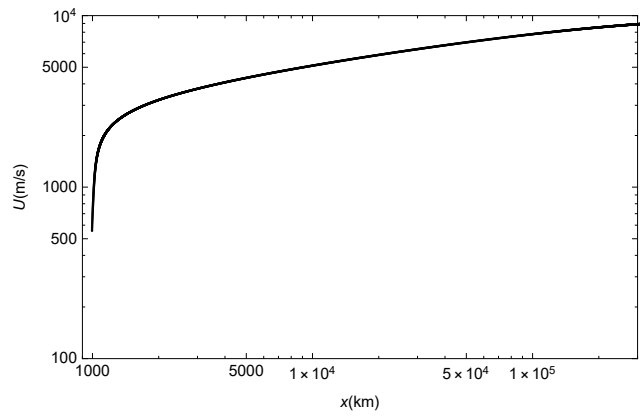

Figure 2: MHD calculation of height dependence of the solar wind velocity profile $U(x)$ in logarithmic scale. The wind profile resembles the temperature profile because $\Theta_{0}$ is the dominating term in the brackets in Eq. (39), while $\tau$ is of the order of one and $\tau \ll \bar{U} \ll \Theta_{0}$.

and what is predicted. That is why there is a calamity of ideas still on the arena, for a contemporary review see the SOHO proceedings ([35]). From qualitative point of view, the narrow width of the TR $\lambda=\min |\mathrm{d} x / \mathrm{d} \ln T(x)|$ is the main property which should be compared against the predictions of other scenarios. For example, in order for the nanoflare hypothesis to be vindicated [36], such reconnections are needed to explain the narrow width of the TR at the same boundary conditions of wind velocity and temperature. Moreover, electric fields of the reconnections heats mainly the electron component of the plasma. How then proton temperature in the corona is higher? Launching of Hinode gave a lot of hints for the existence of AW in the corona [37], see also Ref. [38]. However, most of the those research was in UV region when high frequency AW which heat are already absorbed. All observations are for low frequency (mHz range) AW for which hot corona is transparent. The best can be done is to extract low frequency behavior of the spectral density of AW and to extrapolate to higher frequencies responsible for heating. So observed AW are irrelevant for the heating. In order to identify AW responsible for the heating, it is necessary to investigate high frequency ( $1 \mathrm{~Hz}$ range) AW in the cold chromosphere using optical, not UV spectral lines. We are unaware whether such type of experiments are planned. One of the purposes of the present work is to focus the attention of experimentalists on the $1 \mathrm{~Hz}$ range AW in the chromosphere, which we predict on the basis of our MHD analysis. For such purposes we suggest a special attention to be paid to the behavior of oscillations [39] and sunspot waves [40] above active regions. Another possibility is provided by Doppler tomography [41] of $\mathrm{H} \alpha$ or Ca lines. Doppler tomography was successfully used for investigation of rotating objects, such as accretion disks [42] and solar tornados [43]. Here we wish to mark also the Doppler tomography by Coronal Multi-channel Polarimeter build by Tomczyk [6]. For investigation of AW by Doppler tomography we suggest development of frequency dependent Doppler tomography operating as a lock-in voltmeter. The date from every space pixel should be multiplied by $\sin \omega t$ and integrated for many wave periods. Finally one can observe time averaged distribution of the AW amplitude. Systematic investigation of such frequency dependent Doppler tomograms will reveal that Swedish iconoclast [34] is again right that AW heat the solar corona, after another 70 years of dramatic launching of vast variety of ideas. In this article we wish to emphasize that the concept of self-induced opacity with respect to MHD waves 


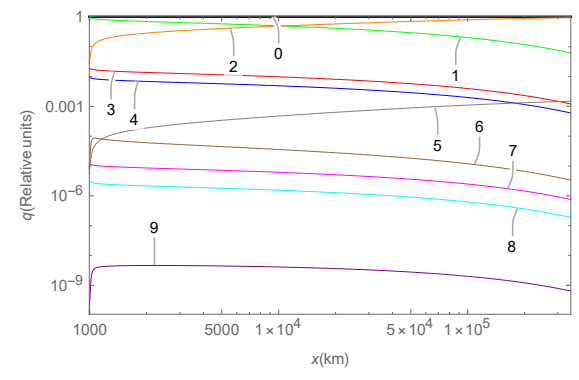

Figure 3: Detailed analysis of all energy flux density terms defined in Eq. (26) and Eq. (27). Height $x$ dependence of the logarithms of the modulus of different components of the energy flux. Labels of curves correspond to: 0 ) the total constant energy flux density $q$, which is integral of motion of MHD equations, 1) the ideal wave energy flux term $q_{u b}$ containing both wave velocity $\hat{u}$ and magnetic field $\hat{b}, 2)$ the wind pressure energy flux term $q_{P}$, 3 ) the ideal wave energy flux term $q_{b}$; for this "aether" term a magnetic energy $\propto|\hat{b}|^{2}$ is moving with the velocity of the wind $U$, 4) the ideal wave velocity energy flux term $\left.q_{u}, 5\right)$ the ideal wind energy flux acceleration term $q_{U}, 6$ ) the wind thermal conductivity dissipative energy flux term $q_{\varkappa}, 7$ ) the wave dissipative viscous energy flux term $q_{\eta}, 8$ ) the wave dissipative Ohmic resistivity flux term $q_{\varrho}$ and 9) the wind viscous dissipative energy flux term $q_{\xi}$. Both wind dissipative terms $q_{\xi}$ and $q_{\varkappa}$ are negative and describe leeward energy flux. All other terms describe windward energy fluxes. Despite being small, $q_{\varkappa}$ and $q_{\xi}$ contain space derivatives and determine the shape of temperature $T(x)$ and wind velocity $U(x)$ profiles.

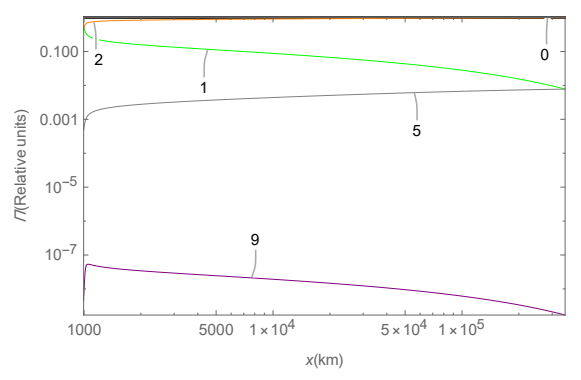

Figure 4: Detailed analysis of all momentum flux density terms defined in Eq. (28) and Eq. (29). Labels of curves correspond to: 0 ) the total constant momentum flux density $\Pi$, which is integral of motion, 1) the wave momentum flux term $\Pi_{\text {wave }}, 2$ ) the wind pressure momentum flux term $\left.\Pi_{P}, 5\right)$ the ideal wind momentum flux acceleration term $\Pi_{U}$ and 9) the dissipative viscous momentum flux term $\Pi_{\xi}$. As for the energy flux, this small term contains space derivative $\mathrm{d} / \mathrm{d} x$ and we have singular perturbation theory for a system of differential equations for the wind variables $T(x)$ Eq. (38) and $U(x)$ Eq. (36).

was qualitatively proposed by us several months before launching the Hinode mission; now we are presenting only the detailed MHD analysis confirming our qualitative consideration ten years ago.

\subsection{Plasma heating by AW - a historical perspective}

What have we learned from the one-dimensional static MHD problem? We have demonstrated that qualitatively predicted self-induced opacity of plasma is an intrinsic property. Absorption of AW causes viscous heating of ions and that is why the proton temperature is higher than the electron one. In this way we have revealed an effective method for ion heating which can be applied to many 


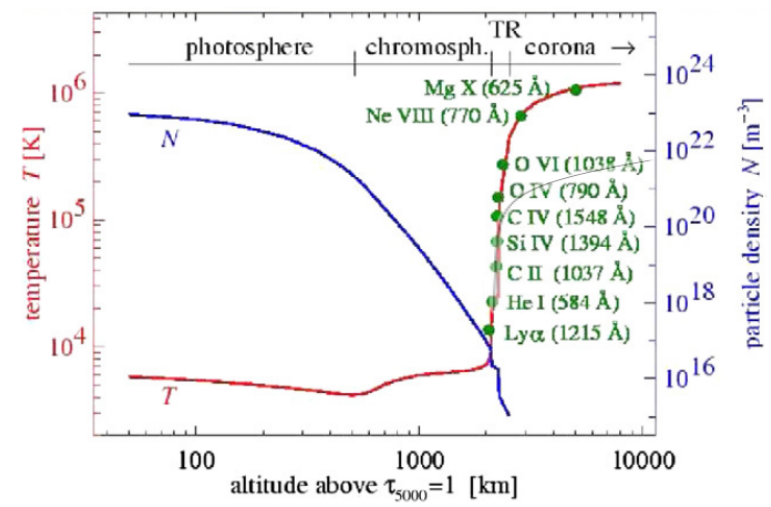

Figure 5: The thin increasing line is the temperature profile $T(x)$ from MHD calculation presented in Fig. 1. This illustrative MHD example is imposed together with observational data of Ref. [13]. The thick line with mark ' $T$ ' is $T(x)$ from Ref. [13], spectral lines of elements reveal how this observational curve is derived; the thick line with mark ' $N$ ' is the particle density $n(x)$ also in logarithmic scale in right ordinate. In the lower part of the TR between $10^{4} \mathrm{~K}$ and $10^{5} \mathrm{~K}$ MHD calculation almost perfectly matches the observational data. For higher temperatures the MHD calculation which contains only one monochromatic AW gives lower temperatures than the experimental profile. Optimistically we believe that MHD calculations with several AW could fit to whole observational $T(x)$.

plasma problems. Actually plasma heating by MHD waves is used in the MIT alcator [44]. We suggest however that the toroidal geometry can be replaced by Budker probkotron geometry [45], in which the energy of the AW will be focused in a narrow jet with a hundred times increased temperature. A de Laval nozzle will be realized by strong magnetic fields. We do believe that this will be an effective method for navigation in the Solar System (cf. [46]). Electric power from a nuclear reactor will create a fast electron-proton jet and this will dramatically decrease the initial mass of the rocket. For large-scale Earth-based installations such a jet of high-temperature deuterium will inject a fresh idea in nuclear fusion physics. For this purposes, however, we need of detailed function of temperature and magnetic field dependence of plasma kinetic coefficients. Such investigations started seventy years ago by R. Landshoff [47], E. S. Fradkin in 1951 [48], I. E. Tamm [49] and S. I. Braginskii in 1952 [50]. The main final results are listed in the Kinetics by Lifshitz and Pitaevskii [23]. Unfortunately we are unaware of recent reviews elaborating the old one by Braginskii [25].

Other unresolved problems are the second viscosity, kinetic coefficients and enthalpy of partially ionized hydrogen plasma. Having approximated experimental data with our calculation of temperature $T(x)$ and wind velocity $U(x)$ profiles, our work can be extended to the chromosphere. In such a way, solving the problem of kinetics and magnetohydrodynamics of solar plasma we can concentrate our attention to solve the inverse problem of revealing the spectral density of MHD waves coming from the invisible layers of the sun.

Our MHD analysis confirmed that the narrow transition region is almost a permanent explosion of chromospheric plasma by self-induced opacity with respect of MHD waves. There is no other scenario able to explain the width of the transition region. Except the thermal expansion of completely ionized plasma, a significant contribution for launching of the solar wind gives intensive low frequency MHD waves reflected from the TR as Fresnel reflection by a jump of the mass density $\rho(x)=\operatorname{Mn}(x)$ and of the corresponding refraction coefficient $\mathcal{N}_{\mathrm{r}}=V_{\mathrm{A}}(0) / V_{\mathrm{A}}(x)=\sqrt{U(0) / U(x)}$. The local Alfvén speed 
$V_{\mathrm{A}}(x)=B_{0} / \sqrt{\mu_{0} \rho(x)}$ also has step-like behavior and the jump of the effective refraction coefficient creates strong reflection too, giving momentum to the TR. Unfortunately, up to now there is no reliable observational data for the height profile the solar wind $U(x)$. Using our routines we will continue MHD analysis in this direction but it will be nice to have alternative considerations. The contribution of low-frequency AW to the launching of solar wind is the next problem which has to be set in the agenda of the astrophysics.

Existence of intensive Alfénic modes in TR can be confirmed by systematic investigation of nonthermal broadening of spectral lines of $\mathrm{Mg}$, and other elements both in the chromosphere and transition region. Moreover, taking into account that magnetic field is more or less vertical with respect to the Sun surface, the transversal AW create horizontal oscillations of the velocity and the transversal Doppler effect will give maximal broadening close to periphery of the solar disk and minimal at the center.

In the present work we concentrated our attention to the transversal to the magnetic field AW. In the chromosphere however, ionization-recombination processes will create a significant second viscosity $\zeta$ and high-frequency slow magnetosonic waves should be absorbed in the chromosphere. For heating of the chromosphere we need to know temperature dependence $\zeta(T)$ and the enthalpy of hydrogen plasma. We are unaware of such papers and will put this task in the agenda for further research.

For our approach, incoming AW are simply boundary condition. AW propagate along the magnetic force lines as through wave-guide. It is now subject of speculations where AW are created by perturbation of the magnetic pipes by turbulent convection or MHD waves are created in the solar tachocline [51] by self-sustained-wave turbulence in shear flow. The last scenario is perhaps working for the jets accompanying accretion [52]. In accretion disks the the effective viscosity and heating is created by self-sustained wave turbulence. Small part of accreting plasma following magnetic force lines is heated and accelerated in jets analogously to the solar wind; this will be a task of space MHD in the next decades.

One dimensional wave-guide approximation can be extended far from the TR to the distances of order of solar radius and even the size of solar system. In this case it is necessary to take into account slow adiabatic increasing of the area of the plasma stream and corresponding decrease of the constant longitudinal magnetic field at approximately constant magnetic flux.

Such type of contribution for the propulsion can be realized in a probkotron used simultaneously as a resonator. In such a way, hydrodynamic approach puts in the agenda many problems which can be easily solved knowing the detailed magnetic and temperature dependence of kinetic coefficients of solar plasma.

The main defect of the present research is that in frequency $\omega$ representation we solve static equations which require sophisticated numerical methods. All those results have to be repeated in time $t$ dependent representation using the brute force of Monte Carlo averaging of standard MHD calculations.

A review of various mechanisms for solar corona heating could be easily made - most surely there is no plasma physical process that has not been used as the long-sought mechanism for solar corona heating. Nevertheless, we will point some alternative scenarios, which according to us even qualitatively contradict to the observations.

The longitudinal magnetosonic waves are intensely absorbed in the chromosphere through the large second viscosity and in practice do not reach the corona. And in reality, the intensity of the longitudinal waves in the solar wind is significantly lower than the Alfvén waves intensity.

If nanoflares and magnetic reconnections are the main mechanism for heating, then plasma energy absorption will be by Ohmic heating of the electrons. However, the proton temperature is higher than 
the electron one in the solar wind [26]. For more thorough review, please see one of the most recent works [32].

According the best we know there are no other attempts for calculation of the temperature profile of TR. In such a way the acceptable agreement between the observational data and our MHD analysis allows to conclude that after 70 years we have reliably returned to Alfvén [2] idea that heating of solar corona is predominantly by AW and the problem of set by Grotrian and Edlen: what heats the solar corona is already solved - 80 years perhaps the most long living problem of the science.

Authors are thankful to Yana Maneva [53-56] and Martin Stoev for the collaboration in the early stages of the present research [14] when the idea of self-induced opacity was advocated and consideration of many problems related to physics of solar corona. Fruitful comments and discussions with Tsvetan Sariisky are highly appreciated.

Authors are also thankful to Ramesh Chandra and Reetika Joshi for discussions of the non-thermal broadening of Mg II lines, Vassil Vassilev for his interest in our work, Ivan Zhelyazkov, Yana Maneva, Steven R. Cranmer, Eckart Marsch, Leon Ofman, Stefaan Poedts, Jaime Araneda, Plamen Angelov, Ramesh Chandra and Yurii Dumin, for short discussions and comments.

\section{References}

[1] P. Swings, Astrophys. J. 98, 116-128 (1943); B. Edlén, Arkiv f. Matem. Astr. och Fys. 28, B No. 1 (1941); B. Edlén, Zs. f. Ap. 22, 30 (1942).

[2] H. Alfvén, MNRAS 107, 211-219 (1947).

[3] H. Alfvén, Nature 150, 405-406 (1942).

[4] J. Heyvaerts and E. R. Priest, A\&A 117, 220 (1983).

[5] L. P. Chitta, A. A. Ballegooijen, L. Rouppe van der Voort, E. E. DeLuca, and R. Kariyappa, Astrophys. J. 752, 48, (2012); http://arxiv.org/abs/1204.4362.

[6] S. Tomczyk and S. W. McIntosh, Asprophys. J; http://arxiv.org/abs/0903.2002.

[7] R. Erdélyi and V. Fedun, Are There Alfvén Waves in the Solar Atmosphere?, Science 318, 1572 1574 (2007);

[8] S. Tomczyk, S. W. McIntosh, S. L. Keil, P. G. Judge, T. Schad, D. H. Seeley, and J. Edmondson, Science 317, 1192 (2007).

[9] G. L. Withbroe and R. W. Noyes, Annual review of astronomy and astrophysics 15, 363-387, (1997), Figure 3.

[10] J. A. Eddy, A New Sun: The Solar Results from Skylab, ed. by R. Ise, prep. by George C. Marshall Space Flight Center, NASA, Washington, D.C., (1979), Fig on page 2, Fig on page 36, https://history.nasa.gov/SP-402/p2.htm.

[11] M. J. Aschwanden, Physics of the Solar Corona. An Introduction with Problems and Solutions (Springer, New York, 2005).

[12] E. H. Avrett and R. Loeser, The Astrophysical Journal Supplement Series 175, Issue 1 p. 229 276, (2008).

[13] H. Tian, E. Marsch, C. Tu, W. Curdt, J. He, New Astronomy Reviews 54, p. 13-30, (2010), Fig. 1 Lower.

[14] T. M. Mishonov, M. V. Stoev, and Y. G. Maneva, Eur. Phys. J. D 44, 533-536 (2007); arXiv:astro-ph/0701908; arXiv:astro-ph/0701554; arXiv:astro-ph/0609609.

[15] T. K. Suzuki, Nonlinear Processes in Geophysics 15, 2, 295-204 (2008).

[16] L. Ofman, Journal of Geophysical Research 115, A4 (2010).

[17] L. Ofman, Space Science Reviews 120, 1-2, 67-94 (2008).

[18] V. M. Nakariakov, L. Ofman, T. D. Arber, Astronomy and Astrophysics 353, 741-748 (2000). 
[19] L. Ofman, J. M. Davila, Journal of Geophysical Research 103, A10, 23677-23690 (1998).

[20] L. Ofman, J. M. Davila, Journal of Geophysical Research 100, A12, 23413-23426 (1995).

[21] S. Vasheghani Farahani et al., Astronomy and Astrophysics 544, A127, 5 (2012).

[22] L. D. Landau and E. M. Lifshitz, Course of Theoretical Physics, Vol. 8, Electrodynamics of Continuous Media, 6th ed. (Pergamon, New York, 1984), Chap. 8 "Magnetohydrodynamics" Eqs. (63.6-8), (65.7-15), (66.2-5,9), sec. 69, Problem, Eq. (69.8).

[23] L. D. Landau and E. M. Lifshitz, Course of Theoretical Physics, Vol. 10, Physical Kinetics, 1st ed. (Nauka, Moscow, 1979),

Sec. 13 "Kinetic phenomena in gases in external field" Eq. (13.18),

Sec. 42 "Energy transfer between electrons and ions" Eqs. $(42.5,6)$,

Sec. 43 "Particle mean free path in plasma" Eqs. (43.4-10),

Sec. 58 "Hydrodynamic equations of magnetoactive plasma" Eq. (58.16),

Sec. 59 "Plasma kinetic coefficients in strong magnetic field" Eq. (59.38).

[24] E. Landi and M. Landini, Astronomy and Astrophysics 347, 401-408, (1999).

[25] S. I. Braginskii, Transport Phenomena in Plasma, Vol. 1 (Atomizdat, Moscow, 1963) (in Russian);

Transport Processes in Plasma, Reviews of Plasma Physics Vol. 1 205, (1965), Eq. (4.44);

E. M. Epperlein and M. G. Haines, "Plasma transport coefficients in a magnetic field by direct numerical solution of Fokker-Planck equation," Physics of Fluids 29, p. 1029 (1986).

[26] L. F. Burlaga and W. H. Mish, J. Gephys. Res., 92, 1261 (1987); E. Marsch, in Physics and Chemistry in Space - Space and Solar Physics, Vol. 21, Series Editors: M. C. E. Huber et al., Physics of the Inner Heliosphere, Vol. 2, Editors: R. Schwenn and E. Marsch, Springer-Verlag Berlin (1991); Fig. 10.4.

[27] R. V. Topchiyska, N. I. Zahariev, T. M. Mishonov, Bulg. J. Phys. 40, 56-77 (2013);

N. I. Zahariev, T. M. Mishonov, AIP Conf. Proc. 1356, 123 (2011);

T. M. Mishonov, N. I. Zahariev, R. V. Topchiyska, B. V. Lazov, S. B. Mladenov, in Proceedings of the Bulgarian National Conference on Physics 2014, Plovdiv, Bulgaria, Bulgarian Chemical Communications, Vol. 47, Special Issue B, pp. 368-379 (2015);

T. M. Mishonov, N. I. Zahariev, R. V. Topchiyska, B. V. Lazov, S. B. Mladenov, A. M. Varonov, https://arxiv.org/abs/1103.2233.

[28] R. P. Feynman, R. B. Leighton, and M. Sands, The Feynman Lectures on Physics. Exercises (Addison-Wesley, London, 1965), Problems 40.4-5.

[29] T. Van Doorsselaere et al., The Astrophysical Journal Letters 727, 2, L32, 4 (2011).

[30] L. D. Landau and E. M. Lifshitz, Course of Theoretical Physics, Vol. 3, Quantum Mechanics, (3 ed., Pergamon, New York, 1977),

Sec. 21, "General properties of one dimensional Schödinger equation.

[31] https://www.encyclopediaofmath.org/index.php/Absorbing_boundary_conditions

J. P. Berenger, "A perfectly matched layer for the absorption of electromagnetic waves"

J. Comp. Phys. 114, 185-200 (1994); C. Cerjan, D. Kosloff, R. Kosloff, M. Reshef, Geophysics 50, 705-708 (1985) ; R. W. Clayton, B. Engquist, Bull. Seis. Soc. Amer. 67, 1529-1540 (1977); B. Engquist, A. Majda, L. Halpern, L. N. Trefethen, J. Acoust. Soc. Amer. 84, 1397-1404 (1988); E. L. Lindman, J. Comp. Phys. 18, 66-78 (1975); IEEE Trans. Electromagn. Compat. 23, 377-382 (1981); R. A. Renaut, J. Comp. Phys. 102, 236-251; R. A. Renaut, J. Fröhlich, J. Comp. Phys. 124, 324-336 (1996); R. A. Renaut, J. Peterson, Geophysics 54, 1153-1163 (1989); A. C. Reynolds, Geophysics 43, 1099-1110 (1978); P.A. Tirkas, C.A. Balanis, R.A. Renaut, IEEE Trans. Antennas and Propagation 40: 10, 1215-1222 (1992). 
[32] T. Sakurai, Proc. Jpn. Acad., Ser. B 93, pp. 87-97 (2017).

[33] W. Grotrian, Naturwissenschaften 27, 214, (1939);

B. Edlén, Z. Physik 104, 407 (1937).

[34] A. J. Dessler, Science 170, 604 - 606 (1970); http://www.sciencemag.org/content/170/3958/604.

[35] Proceedings of the SOHO 15 Workshop - Coronal Heating, 6-9 September 2004, St. Andrews, Scotland (ESA SP-575, December 2004) Editors: R. W. Walsh, J. Ireland, D. Danesy, and B. Fleck.

[36] Ch. Day, Phys. Today 62, May 18-21 (2009).

[37] B. De Pontieu, et al., Science 318, 1574-1577 (2007); T.J. Okamoto et al., Science 318, 15771580 (2007); Y. Katsukawa et al., Science 318, 1594-1597 (2007).

[38] D. Jess et al., Science 323, 1582-1585 (2009).

[39] D. Y. Kolobov, N. I. Kobanov, A. A. Chelpanov, A. A. Kochanov, S. A. Anfinogentov, S. A. Chupin, I. I. Myshyakov, V. E. Tomin, Advances in Space Research 56, pp. 2760-2768 (2015).

[40] R. Chandra, G. R. Gupta, Sargam Mulay and Durgesh Tripathi, MNRAS 446, pp. 3741-3748 (2015).

[41] T. Marsh, Europhysicsnews 36 No. 4, 133-138 (2005).

[42] T. R. Marsh and K. Horn, MNRAS 235, 269-286 (1988).

[43] http://www.mps.mpg.de/en/forschung/sonne/.

[44] J. A. Snipes et al., Phys. Plasmas 12, 056102 (2005); http://dx.doi.org/10.1063/1.1865012.

[45] N. A. Krall and A. W. Trivelpiece, Principles of Plasma Physics, (McGraw-Hill, New York, 1973); Figs. 92, 101, 102, 104, 107.

[46] E. Y. Choueiri, Scientific American 300, 58-65 (2009); doi:10.1038/scientificamerican0209-58.

[47] R. Landshoff, Phys. Rev. 76, p. 904 (1949).

[48] E. S. Fradkin, JETP 5, No. 5, p. 956 (1957); Report FIAN, unpublished (1950-1951) (in Russian).

[49] I. E. Tamm, A. D. Sakharov, Physica Institute of the Academy of Sciences of U.S.S.R. Otchety (Russian title: Otchety o magnitnom termoyadernykh reaktorakh) (1951) (in Russian).

[50] S. I. Braginskii, ZhETF 33, No. 3, p. 645, (1957) (in Russian);

JETP 6, No. 3, p. 494 (1958) (English translation);

p. 494, Footnote: "This work was performed in 1952."

[51] https://en.wikipedia.org/wiki/Tachocline;

E. A. Spiegel, J.-P. Zahn, Astronomy and Astrophysics 265, no 1, 106-114;

P. Charbonneau et al, The Astrophys. J. 527, Issue 1, pp. 445-460 (1999).

[52] https://en.wikipedia.org/wiki/Astrophysical_jet;

I. Ciufolini and J. Wheeler, Gravitation and Inertia, (Princeton University Press, Princeton, New Jersey, 1995), Sec. 6.3, Pictures 4.5, 6.1-4;

Speical issue of the Astrophysical Journal Letters dedicated to the Hubble Space Telescope 435;

H. C. Ford et al., Astrophys. J. Lett. 435, L27-L30 (1994);

R. J. Harms et al., Astrophys. J. Lett. 435, L35-L38 (1994);

R. D. Blandford, R. L. Znajek, MNRAS, 179, Issue 3, p. 433-456, (1977).

[53] J. A. Araneda, Y. Maneva, and E. Marsch, Phys. Rev. Lett. 102, 175001 (2009).

[54] Y. G. Maneva, J. A. Araneda, and E. Marsch, ed. by I. Zhelyazkov, AIP Conference Proceedings, vol. 1121, pp. 122-126 (2009).

[55] Y. G. Maneva, J. A. Araneda, and E. Marsch, Twelfth International Solar Wind Conference, eds. M. Maksimovic, K. Issautier, N. Meyer-Vernet, M. Moncuquet and F. Pantellini, AIP Conference 
Proceedings, vol. 1216, pp. 227-230, (2010).

[56] Y. G. Maneva, Ph.D. Thesis, (Göttingen, uni-edition, 2010), ISBN 978-3-942171-39-7.

[57] G. A. Baker Jr. and P. Graves-Morris, Padé Approximants, Second edition, (Cambridge Univ. Press, Cambridge 1996).

[58] L. D. Landau and E. M. Lifshitz, Course of Theoretical Physics, Vol. 5, Statistical Physics, Part 1, (3 ed., Pergamon, New York, 1977),

Eq. (11.4), Eq. (15.12) "Theorem of small corrections", Sec. 78 "Thermodynamic variables of classical plasma”, Eqs. (78.11-14), Sec. 79 "Method of correlation functions" Sec. 104 "Ionization equilibrium" Eqs. (104.4,5).

[59] A. B. Migdal, Qualitative methods in quantum mechanics (Moskow, Nauka, 1975) (in Russian), Chapter 1, "Dimensional and model evaluations".

[60] L. D. Landau and E. M. Lifshitz, Course of Theoretical Physics, Vol. 2, The classical Theory of field, 7th ed. (Pergamon, New York, 1989),

Sec. 33 "Tensor energy-momentum of electromagnetic field" Eqs. (33.3), (33.9),

Sec. 68 "Dipole emission at scattering", Eq (68.1), Sec. 70 "Radiation in the case of Coulomb interaction", Eq. (70.22), Prob. 2 and 4.

[61] L. D. Landau and E. M. Lifshitz, Course of Theoretical Physics, Vol. 4, V. B. Beresteckii, L. D. Landau and E. M. Lifshitz Quantum electrodynamics, (3 ed., Pergamon, New York, 1990), Sec. 92, "Electron-nucleus bremsstrahlung. The non-relativistic case ", Eq. (92.23), Problems 2 and 5.

[62] L. D. Landau and E. M. Lifshitz, Course of Theoretical Physics, Vol. 6, Hydrodynamics, 4th ed. (Pergamon, New York, 1985), Chap. 8 "Viscous liquid" Sec. 15 "Equation of motion of viscous liquid" Eq. (15.6).

[63] L. D. Landau and E. M. Lifshitz, Course of Theoretical Physics, E. M. Lifshitz and L. P. Pitaevskii Vol. 9, Statistical Physics, Part 2, Chap. VIII, "Electromagnetic fluctuations" Sec. 85, "Degenerated plasma", Eq. (85.23-24) (Pergamon, New York, 1979),

[64] L. D. Landau and E. M. Lifshitz, Course of Theoretical Physics, Vol. 1, Mechanics, (Pergamon, New York, 1989),

Sec. 40, "Hamilton equations".

[65] L. D. Landau and E. M. Lifshitz, Course of Theoretical Physics, Vol. 7, Theory of elasticity, (Pergamon, New York, 1989),

Sec. "Strength tensor" Eq. (2.1).

\section{A Elementary statistics and kinetics of hydrogen plasma}

In this section following the kinetics by Lifshitz and Pitaevskii [23], we will repeat the gas kinetic formulas applied to the plasma. Let us begin with the thermal velocities of the electrons and the protons

$$
v_{T e} \equiv \sqrt{T_{e} / m}, \quad v_{T p} \equiv \sqrt{T_{p} / M},
$$

and introduce the variable $r_{T} \equiv e^{2} / T$ with length dimension. The electron and proton transport sections for the Coulomb interaction expressed with the new variable have the form

$$
\sigma_{e e}=\frac{\Lambda}{0.6} r_{T}^{2}, \quad \sigma_{p p}=\frac{\Lambda}{0.4} r_{T}^{2}, \quad \Lambda=\ln \frac{r_{\mathrm{D}}}{r_{T}} .
$$

For Rutherford scattering the cross-section is infinite and in the precise theory this infinity is substituted by the Coulomb logarithm from a big number: the ratio of the Debye radius $r_{\mathrm{D}}$ and the smallest 
distance $r_{T}$ between two protons with thermal energy $T$. The coefficients 0.6 and 0.4 are added to the final exact formulas for respectively the electric conductivity and viscosity coefficients of completely ionized hydrogen plasma.

In a gaseous approximation the reciprocal mean free path for one electron is additive

$$
\frac{1}{l_{e}}=\sigma_{e e} n_{e}+\sigma_{e a} n_{a}
$$

where $\sigma_{e a}$ is the electron-atom cross section and $n_{a}$ is the atom density. The mean free path $l_{e}$ is connected with the mean free time

$$
\tau_{e}=\frac{l_{e}}{v_{T e}}=\frac{0.6}{\Lambda} \frac{m^{1 / 2} T^{3 / 2}}{e^{4} n_{e}},
$$

and the collision rate $v_{e}=1 / \tau_{e}$. The elementary kinetic estimate for the electric conductivity $\varsigma$ and the ohmic resistivity $\varrho[23]$ connected with it

$$
\varsigma=\frac{1}{\varrho}=n_{e} q_{e}^{2} \frac{\tau_{e}}{m}
$$

gives for the magnetic field diffusion coefficient

$$
\nu_{\mathrm{m}}=\varepsilon_{0} c^{2} \varrho=\frac{c^{2}}{4 \pi} \frac{m}{e^{2} n_{e} \tau_{e}}=\frac{c^{2}}{4 \pi} \frac{\sqrt{m T_{e}}}{e^{2} n_{e}}\left(n_{e} \frac{\Lambda}{0.6} r_{T}^{2}+n_{a} \sigma_{e a}\right) .
$$

At high temperatures $n_{a} \rightarrow 0$ this formula gives the well known result for the Ohmic resistance and electric conductivity of fully ionized hydrogen plasma

$$
\varrho=\frac{1}{\varsigma}=\frac{1}{4 \pi \epsilon_{0}} \frac{\Lambda}{0.6} \frac{e^{2} m^{1 / 2}}{T^{3 / 2}}, \quad \frac{\varsigma}{4 \pi \varepsilon_{0}}=0.6 \frac{T^{3 / 2}}{e^{2} m^{1 / 2} \Lambda} .
$$

In such a way, the approximate estimation for the transport section Eq. (64) exactly reproduces the well-known result [23].

Analogously, the heat conductivity coefficient is determined by the heat capacity per one electron $c_{e}=\frac{3}{2}$ and the elementary kinetic estimate

$$
\varkappa=c_{e} n_{e} l_{e} v_{T e}
$$

gives

$$
\varkappa=\frac{3}{2} n_{e} \sqrt{\frac{T_{e}}{m}}\left(n_{e} \frac{\Lambda}{0.6} r_{T}^{2}+n_{a} \sigma_{e a}\right)^{-1} .
$$

For completely ionized hydrogen plasma the heat conductivity coefficient becomes

$$
\varkappa=\frac{0.9}{\Lambda} \frac{T^{5 / 2}}{e^{4} m^{1 / 2}}
$$

Similarly, for the reciprocal proton mean free path we have

$$
\frac{1}{l_{p}}=n_{p} \sigma_{p p}+n_{a} \sigma_{p a}
$$


where $\sigma_{p a}$ is the proton-atom cross section. The elementary evaluation for the kinetic viscosity gives

$$
v_{\mathrm{k}}=l_{p} v_{T_{p}}=\sqrt{\frac{T_{p}}{M}}\left(n_{p} \frac{\Lambda_{p}}{0.4} r_{T}^{2}+n_{a} \sigma_{p a}\right)^{-1}
$$

and for fully ionized plasma we arrive at the well known result for the viscosity Eq. (43.10) of Ref. [23]

$$
\eta=\rho v_{\mathrm{k}}=\frac{0.4}{\Lambda} \frac{T_{p}^{5 / 2} M^{1 / 2}}{e^{4}}
$$

Let us also mention the following relations between the kinetic coefficients

$$
\begin{aligned}
& \varkappa \varrho=1.5 T_{e} / q_{e}^{2}, \\
& \eta / \varkappa \approx \frac{4}{9} \sqrt{m M} .
\end{aligned}
$$

For the temperature dependent magnetic Prandtl coefficient $\mathrm{P}_{\mathrm{m}} \equiv v_{\mathrm{k}} / v_{\mathrm{m}}$ we have

$$
\operatorname{Pr}_{\mathrm{m}}=\frac{4 \pi e^{2} n_{e}}{c^{2} \sqrt{m M}}\left[\left(n_{e} \frac{\Lambda r_{T}^{2}}{0.6}+n_{a} \sigma_{e a}\right)\left(n_{p} \frac{\Lambda r_{T}^{2}}{0.4}+n_{a} \sigma_{p a}\right)\right]^{-1},
$$

and for high temperatures when $n_{a} \rightarrow 0$ we have

$$
\operatorname{Pr}_{\mathrm{m}}=\frac{0.96 \pi}{\Lambda^{2}} \frac{T^{4}}{\sqrt{m M} c^{2} e^{6} n} \gg 1 ;
$$

i.e. the electric resistance is negligible. While for the cold chromosphere the magnetic Prandtl number is of order of one, for the hot corona with a 100 times higher $T$ it increases by 8 orders of magnitude. For this reason, in the whole interval where we consider MHD heating, the coefficient $v_{\mathrm{m}}$ is negligible. In other words, the formal inclusion of Ohmic heating in the MHD equations will not change the profile $T(x)$ in the range of highest temperatures which is exactly the purpose of our consideration. Therefore, for illustrative purposes, we can take $v_{\mathrm{m}}=0$. While for low temperatures in the chromosphere, it is possible to neglect the viscosity and the heating could be purely ohmic.

Let us give an elementary estimate for ratios of the corresponding times and collision rates for electron, proton and electron-proton collisions for energy exchange $\tau_{e p}^{\varepsilon}$ for completely ionized hydrogen plasma

$$
\left(\tau_{e}=\frac{1}{v_{e}}\right):\left(\tau_{p}=\frac{1}{v_{p}}\right):\left(\tau_{e p}^{\varepsilon}=\frac{1}{v_{e p}^{\varepsilon}}\right)=1: \sqrt{\frac{M}{m}}: \frac{M}{m} .
$$

In the last proportion, it is taken into account that for elastic electron proton collision the exchange of energy is of order of $m / M$; it can be derived considering the scattering in the system of center of the mass. Our elementary consideration $\tau_{e p}^{\varepsilon}=\tau_{e}(M / m)$ gives

$$
\tau_{e p}^{\varepsilon}=\frac{1}{8 \sqrt{2 \pi}} \frac{T^{3 / 2} M}{e^{4} n \Lambda m^{1 / 2}},
$$

where the numerical coefficient $8 \sqrt{2 \pi} \approx 20$ in Eq. (42.5) of Ref. [23] requires state-of-the-art consideration. The kinetic of heat exchange between protons and electrons

$$
\frac{\mathrm{d} T_{e}}{\mathrm{~d} t}=-\frac{T_{e}-T_{p}}{\tau_{e p}^{\varepsilon}}
$$


shows that in the transition region with width $\lambda$ we can consider equal temperatures $T_{e}=T_{p}=T$ if the flight time through the transition region with wind velocity $U$ is much longer than the time for which the protons heat up the electrons

$$
\frac{\lambda}{U} \gg \tau_{e p}^{\varepsilon}
$$

In this way, for hydrogen plasma with known density $\rho$ and temperature $T$, we can determine the pressure $P$ and the kinetic coefficients $\varsigma, \varkappa, \eta$ necessary for our hydrodynamic analysis. For applicability of usual hydrodynamic approach the inequality

$$
v_{p}=\frac{\Lambda}{0.4} \frac{e^{4} n}{T^{3 / 2} M^{1 / 2}} \gg \omega_{B p}=\frac{q_{e} B}{M}, \quad B=H / c
$$

holds perfectly for the chromosphere and the lower boundary of the TR. In the upper boundary of transition layer, i.e. in the beginning of the corona, this inequality is already broken because the density $n$ decreases hundred times and simultaneously $T^{3 / 2}$ increases thousand times.

In order to take into account the influence of the magnetic field on the viscosity for transversal equations for the wave amplitudes in the present paper we suggest the Padé approximant

$$
\eta_{2}=\frac{1}{1+C\left(\omega_{B p} \tau_{p p}\right)^{2}}, \quad C \approx 0.9
$$

which has the same magnetic field dependence as longitudinal electric conductivity in perpendicular magnetic field in $\tau$-approximation. For strong magnetic fields the suggested Padé approximant reproduces the well-known result

$$
\eta_{2}(B \rightarrow \infty) \approx \frac{8 \pi^{1 / 2} e^{4} \Lambda n_{p}^{2}}{5(M T)^{1 / 2} \omega_{B p}^{2}}
$$

for $\omega_{B p} \gg v_{p}$ case Eq. (X.59.38) of Ref. [23]. For all other coefficients $\eta_{1}-\eta_{4}$ we can suggest analogous formulas but the simplest Padé approximation [57] even quantitatively reproduces the main effect of the magnetic field at high temperatures and small densities - viscous heating stops in the hot corona.

\section{B lonization degree}

The ionization degree $\alpha_{l} \equiv n_{p} /\left(n_{p}+n_{a}\right)$ satisfies the Saha equation [58]

$$
\alpha_{I}=1 / \sqrt{1+\left(1+\alpha_{I}\right) R_{n}(T)}
$$

where

$$
R_{n}=\frac{n_{\rho}}{\mathrm{e}^{-I / T} n_{q}}, \quad n_{q}=\left(\frac{m T}{2 \pi \hbar^{2}}\right)^{3 / 2}, \quad I=\frac{1}{2} m c^{2} \alpha_{\mathrm{s}}^{2}, \quad \alpha_{\mathrm{s}}=\frac{e^{2}}{\hbar c} .
$$

Numerical calculation of the Saha equation requires several Newton iterations

$$
\alpha_{I}^{(\text {new })}=\alpha_{I}-\frac{F\left(\alpha_{I}\right)}{\mathrm{d} F / \mathrm{d} \alpha_{I}}
$$

for the function

$$
F\left(\alpha_{I}\right) \equiv \alpha_{I}^{2}-\frac{1}{1+\left(1+\alpha_{I}\right) R_{n}(T)}=0
$$


starting from $\alpha_{I}=1 / 2$.

We also note that protons determine the mass density

$$
n_{\rho}=n_{p}+n_{a}=n_{p} / \alpha_{I}
$$

and then for the pressure we have

$$
P=n_{\mathrm{tot}} T, \quad n_{\mathrm{tot}}=n_{e}+n_{p}+n_{a}=\left(1+\alpha_{I}\right) n_{\rho} .
$$

Ionization degree is important ingredient for the physics of the chromosphere. However, for the temperature of $10 \mathrm{kK}$ and the low density of the upper chromosphere below the TR, the hydrogen is almost completely ionized. Since our calculation starting point is just before the TR, it is justified to assume full ionization $\alpha_{I}=1$.

Knowing total cross-section of ionization by electron impact upon atom $\sigma_{\text {ion }}$ we can evaluate the ionization rate and make elementary evaluation of second viscous coefficient of weakly ionized hydrogen plasma in the chromosphere. Large second viscosity in the chromosphere leads to significant damping of the MHD waves having pressure oscillations. That is why in the TR we concentrate our attention on AW.

\section{Bremsstrahlung}

In this section we will make a model evaluation of the order of the bremsstrahlung in the TR. We follow the style of model evaluations by Migdal [59] Our staring point is the total radiation energy. The total radiation for the collision of electron and proton is [60], cf. also [61].

In the chromospheric boundary of the TR the temperature is low and electron thermal velocity $v_{T e}=\sqrt{T / m}$ is much lower than the Bohr velocity $v_{\mathrm{B}}=e^{2} / \hbar$. For small relative velocities of the charged particles $v_{0} \ll v_{\mathrm{B}}$ we have to use classical electrodynamics for calculation of the energy $\Delta \mathcal{E}$ of radiation emission. For the hydrogen plasma this means that temperature should be lower than atomic energy $1 \mathrm{Ry}=13.6 \mathrm{eV}=158 \mathrm{kK}$. Let us recall the well-known result for the back scattering of two charged particles [60]

$$
\Delta \mathcal{E}(0) \equiv \frac{8 \mu^{3} v^{5}}{45 c^{3} e^{2}}\left(\frac{e}{m}-\frac{e}{M}\right)^{2} \approx \frac{8}{45}\left(m v^{2}\right)\left(\frac{v}{c}\right)^{3},
$$

where $\mu=m M /(m+M)$ is the reduced mass. We are applying this formula for electrons and protons with equal charges and $m \ll M$. With electron energy and the constant of Coulomb interaction we can construct a parameter with dimension of length $r_{0}=e^{2} / \mu v_{0}^{2}$ and to make a model evaluation for the cross-section for energy loss by radiation (effective radiation)

$$
\varkappa=\int_{0}^{\infty} \Delta \mathcal{E}(\rho) \cdot 2 \pi \rho \mathrm{d} \rho \sim \pi r_{0}^{2} \cdot \mathcal{E}(0)=C_{\mathrm{BS}} \frac{e^{4} v_{0}}{c^{3} m},
$$

where $C_{\mathrm{BS}}$ is a dimensionless constant of the order of one. This evaluation agrees with the exact result for the repulsing particles

$$
\varkappa=\frac{8 \pi}{9} \frac{\left|e_{1} e_{2}\right| \mu v_{0}}{c^{3}}\left(\frac{e_{1}}{m_{1}}-\frac{e_{2}}{m_{2}}\right)^{2}, \quad \mu=\frac{m_{1} m_{2}}{m_{1}+m_{2}} .
$$

For the hot Corona where $v_{T e} \gg v_{\mathrm{B}}$ we have to use the results of the quantum electrodynamics [61]. For two electrons, for example, the dipole emission is zero and the quadrupole emission gives

$$
\varkappa=\hbar \int_{0}^{\varepsilon} \omega \mathrm{d} \omega=8.1 \frac{e^{2}}{\hbar c} r_{e}^{2} \varepsilon, \quad r_{e}=\frac{e^{2}}{m c^{2}}, \quad \varepsilon=\frac{1}{2} m v_{0}^{2}, \quad v_{0} \ll c .
$$


We are giving also the result emission of radiation by elastic scattering of a slow $v \ll v_{\mathrm{B}}$ electron by a neutral atom [61]

$$
\varkappa=\int \hbar \omega \mathrm{d} \omega=\frac{32}{45 \pi} \frac{e^{2}}{\hbar c} \sigma_{\text {elast }}\left(\frac{v}{c}\right)^{2}\left(\frac{1}{2} m v^{2}\right) .
$$

In our model qualitative consideration we will substitute the relative velocity by thermal velocity $v_{0} \rightarrow v_{T e}$. We hope that real integration of the corresponding statistical problem gives only multipliers of the order of one. For one scattering center the power of the emitted radiation can be evaluated as averaged flux of particles times the energy loss for scattering $v_{T e} n \sigma \Delta \mathcal{E}=v_{T e} n \varkappa$. For the total volume density of the emitted bremsstrahlung radiation $\tilde{Q}_{\mathrm{BS}}$ we have to multiply to the density of scattering centers

$$
\tilde{Q}_{\mathrm{BS}}=(n v)\left(\Delta \mathcal{E} \sigma_{e p}\right) n=n^{2} v_{\mathrm{Te}} \varkappa .
$$

Substituting here

$$
\sigma_{e p}=\pi r_{T}^{2}=\pi\left(\frac{e^{2}}{T}\right)^{2}, \quad v=v_{T e}
$$

or directly the model evaluation of classical bremsstrahlung evaluation Eq. (91) we arrive at

$$
\tilde{Q}_{\mathrm{BS}}(x)=\frac{8 \pi}{45}\left(\frac{e^{2}}{m c^{2}}\right)^{2} n^{2}(x) c T(x) .
$$

Let us follow a fluid particle moved by the wind with velocity $U(x)$. Integrating the power in time integral $\mathrm{d} t=\mathrm{d} x / U(x)$ we conclude that total energy loss per unit area in interval $(0, x)$ is

$$
\int_{0}^{x} \tilde{Q}_{\mathrm{BS}} \frac{\mathrm{d} x^{\prime}}{U\left(x^{\prime}\right)}
$$

This means that energy flux is already not a constant and for the decreasing of the energy flux we obtain

$$
\Delta q_{\mathrm{BS}}(x)=-U(x) \int_{0}^{x} \tilde{Q}_{\mathrm{BS}} \frac{\mathrm{d} x^{\prime}}{U\left(x^{\prime}\right)} .
$$

Finally, the dimensionless energy flux in the units used for the velocity Eq. (36) and temperature Eq. (38) we derive the term corresponding to the bremsstrahlung

$$
\chi_{\mathrm{BS}}=\frac{\Delta q_{\mathrm{BS}}(x)}{\rho_{0} U_{0}^{3}}=-\frac{U(x)}{\rho_{0} U_{0}^{3}} \int_{0}^{x} \tilde{Q}_{\mathrm{BS}} \frac{\mathrm{d} x^{\prime}}{U\left(x^{\prime}\right)} .
$$

The negative sign corresponds to energy losses. For electron temperatures of order of $1 \mathrm{Ry}$ and higher, the quantum electrodynamics result has a fine constant $\alpha_{\mathrm{s}}$ [61] multiplier and as a result Bremsstrahlung is negligible for a cooling mechanisms in TR.

\section{Gravity}

Since our calculation does not extend beyond 1 solar radius, for the gravitational energy flux we can use the approximate form of the gravitational potential

$$
\mathbf{q}^{\text {grav }}=\rho \mathbf{v} \Phi, \quad \Phi=g_{\odot} x+\text { const }, \quad g_{\odot}>0,
$$

where $g_{\odot}$ is the solar gravitational acceleration. The gravitational energy flux in our notations becomes

$$
\tilde{q}(x)^{\text {grav }}=j g_{\odot} x,
$$


and according to definitions Eq. (32) can be easily expressed in our dimensionless variables

$$
\chi_{\text {grav }}=-\frac{j g_{\odot} x}{\rho_{0} U_{0}^{3}} .
$$

Analogously for the momentum the integrating in one dimensional case the density of gravitational force $f_{g}=-\rho(x) \mathrm{d}_{x} \Phi(x)=-\rho(x) g_{\odot}$ according to momentum conservation law Eq. (3) gives

$$
\tilde{\Pi}_{x x}^{\text {(grav) }}(x)=-\int_{0}^{x} f_{g}\left(x^{\prime}\right) d x^{\prime}=\int_{0}^{x} \rho\left(x^{\prime}\right) g_{\odot} \mathrm{d} x^{\prime} .
$$

Similarly, the momentum flux in dimensionless units becomes

$$
\tau_{\text {grav }}(x)=\left.\frac{\tilde{\Pi}^{\text {grav }}(x)}{\rho_{0} U_{0}^{2}}\right|_{x} ^{0}=-\frac{g_{\odot}}{\rho_{0} U_{0}^{2}} \int_{0}^{x} \rho\left(x^{\prime}\right) \mathrm{d} x^{\prime}
$$

The negative sign of $\tau_{\text {grav }}$ means that gravitational forces decrease the momentum density of solar wind.

The derived dimensionless energy and momentum gravitational fluxes could be easily added to the calculation, or can be evaluated as a perturbation to the calculated solution. Because both of these fluxes have negligible contribution in our region of interest [20], we have not included them in the numerical example analyzed in the present work.

Moreover, taking into account the TR width $\lambda \sim 100 \mathrm{~km}$ and the temperature in its chromospheric end at $T=10 \mathrm{kK}$ for the worst case scenario, we get $M g_{\odot} \lambda / k_{\mathrm{B}} T^{\prime} \approx 3 \times 10^{-2} \ll 1$, i.e. gravity is a negligible perturbation in the TR.

\section{E Momentum equation and distribution of the temperature}

After some algebra following Eq. (15.5) of Ref. [62],

Eq. (33.9) of Ref. [60] and Eq. (66.2) of Ref. [22] Eq. (3) can be rewritten as

$$
\rho\left(\partial_{t}+\mathbf{v} \cdot \nabla\right) \mathbf{v}=-\nabla p+\eta \Delta \mathbf{v}+\left(\zeta+\frac{\eta}{3}\right) \operatorname{grad} \operatorname{div} \mathbf{v}+(\nabla \eta) \cdot \nabla \mathbf{v}+(\nabla \mathbf{v}) \cdot \nabla \eta+(\operatorname{div} \mathbf{v}) \nabla\left(\zeta-\frac{2}{3} \eta\right)-\frac{\operatorname{rot} \mathbf{B}}{\mu_{0}} \times \mathbf{B}
$$

This equation can be derived as $x$ derivative of the constant momentum flux Eq. (28) and different terms give the different densities of the external force.

Quite analogously differentiation of the energy flux Eq. (26) can be derived as the consequence of the equation of heat transport

$$
\rho T\left(\partial_{t}+\mathbf{v} \cdot \nabla\right) s=\operatorname{div}(\varkappa \nabla T)+\sum_{i, k}\left[\eta\left(\partial_{k} v_{i}+\partial_{i} v_{k}-\frac{2}{3} \delta_{i k} \operatorname{div} \mathbf{v}\right)+\zeta \delta_{i k} \operatorname{div} \mathbf{v}\right] \partial_{k} v_{i} .
$$

For an ideal fluid with negligible kinetic coefficients $\eta, \zeta$ and $\varrho$ all terms of this equation are zero.

It is possible to check that upper equation for entropy transport and production is mathematically equivalent to the equation for the conservation of energy Eq. (2). In this sense Eq. (2) can be considered as a proof the evolution equation of the magnetic field Eq. (14). That is why one can say the the complete set of MHD equations Eq. (14) and Eq. (15) can be considered as consequence of mass Eq. (1), energy Eq. (2), and momentum Eq. (3) conservation equations, and the equation of the temperature field $T(\mathbf{r}, t)$ is is also consequence of the conservation equations. 


\section{F Numerical methods}

The differential equations we related to MHD analysis of solar corona are stiff and for them we have to apply the implicit Euler equation in the simplest case

$$
\begin{aligned}
& \mathrm{d}_{x} y=f(x, y), \quad \frac{y(x+h)-y(x)}{h}=f(x+h, y(x+h)), \\
& y=G\left(x, \mathrm{~d}_{x} y\right), \quad y(x+h)=G\left(x+h, \frac{y(x+h)-y(x)}{h}\right), \quad \mathrm{d}_{x} y=\frac{\mathrm{d} y}{\mathrm{~d} x} .
\end{aligned}
$$

The wind variables $y=(T, U)$, and the conservation laws Eq. (26) and Eq. (28) give the possibility to express their derivatives explicitly. We however used the explicit formulas for wind variables $(T(x), U(x))$ : Eq. (36) and Eq. (38) expressed by the derivatives $\left(\mathrm{d}_{x} T(x), \mathrm{d}_{x} U(x)\right)$.

For the wave variables the corresponding system of equations Eq. (22) is linear, formally in the equation above $f(x, y)=\mathrm{A}(x) y$, and the realization of the Euler method reads

$$
y(x+h)=(1-h \mathrm{~A})^{-1} y(h) .
$$

Let $\alpha_{\mathrm{L}}(x)$, and $\alpha_{\mathrm{R}}(x)$, are the smallest eigenvalues of matrix $\mathrm{A}$ describing left- and right propagating waves giving the solution we need. And $\alpha_{\mathrm{D}}(x)$ and $\alpha_{\mathrm{D}^{\prime}}(x)$ are the big in modulus eigenvalues of the parasitic diffusion modes. The implicit Euler method can work in the case of

$$
h\left|\alpha_{\mathrm{L}}\right|, h\left|\alpha_{\mathrm{R}}\right| \ll 1 \ll h\left|\alpha_{\mathrm{D}}\right|, h\left|\alpha_{\mathrm{D}^{\prime}}\right| .
$$

A few words we have to say for the numerical determination of limes. Let $s_{0}, s_{1}, s_{2}, \ldots$ are the values of $\mathrm{y}(\mathrm{x}+\mathrm{h})$ calculated by steps $h, h / 2^{1}, h / 2^{2}, h / 2^{3}, \ldots$ We can apply $\varepsilon$-algorithm for the calculation of $y(x+h)$ as the limes of the sequence $\left\{s_{0}, s_{1}, s_{2}, s_{3}, \ldots\right\}$. In such a way, from the Euler method we can build a high-order method using Padé approximants. Analogously, let the sequence $y_{0}$, $y_{1}, y_{2}, \ldots$ is the Aitken polynomial extrapolation of $y(x+h)$ calculated using $0,1,2,3, \ldots$ interpolation points. Applying again the epsilon algorithm we arrive at $N$-point Padé approximant predictor method for solving system of differential equation; according to the best we know such an opportunity is not used up to now. The $\varepsilon$-algorithm is fast convergent and the optimal order is determined by the error of truncation, i.e. our method has an adaptive order. In such a way, in spite of time consuming, we have a precision method for solving system of ordinary differential equations, which utilizes all numerical resources at a fixed machine precision. Details of the numerical realization of $\varepsilon$-algorithm and will be given in a separate publication.

\section{G Influence of the magnetic field on the kinetic coefficients}

The influence of magnetic field on the kinetic coefficients is significant for the solar corona, but for the TR it is also important to be taken into account. Unfortunately it is not standard for the MHD considerations of the solar corona and that is why the full set of equations is given only in this appendix. Let us start our consideration with the tensor of magnetic viscosity in the Drude $\tau$ approximation for the electron drift velocity $\mathbf{V}_{\mathrm{dr}}$

$$
m \mathrm{~d}_{t} \mathbf{V}_{\mathrm{dr}}=-\frac{m}{\tau_{e e}} \mathbf{V}_{\mathrm{dr}}+q_{e}\left(\mathbf{E}+\mathbf{V}_{\mathrm{dr}} \times \mathbf{B}\right)
$$

and corresponding tensors for the conductivity $\hat{\sigma}$, resistivity $\hat{\varrho}_{\Omega}$ and magnetic diffusivity $\hat{v}_{\mathrm{m}}$

$$
\mathbf{j}=n_{e} q_{e} \mathbf{V}_{\mathrm{dr}}=\hat{\sigma} \mathbf{E}, \quad \mathbf{E}=\hat{\varrho}_{\Omega} \mathbf{j}, \quad \hat{v}_{\mathrm{m}} \equiv \varepsilon_{0} c^{2} \hat{\varrho}_{\Omega}
$$


In the static limit for frequencies $\omega \ll \omega_{B e}$ much lower than the electron cyclotron frequency

$$
\omega_{B e}=\frac{q_{e} B}{m}
$$

For the magnetic diffusivity tensor in the plane $(y, z)$ perpendicular to the constant magnetic field $\mathbf{B}_{0}=B_{0} \mathbf{e}_{x}$ we have

$$
\hat{v}_{\mathrm{m}}=\left(\begin{array}{cc}
1 & -\omega_{B e} \tau_{e e} \\
+\omega_{B e} \tau_{e e} & 1
\end{array}\right) v_{\mathrm{m}}
$$

and the equation for the magnetic field of the AW oscillations Eq. (20) now is reduced to the system

$$
\begin{aligned}
& -\mathrm{i} \omega \hat{b}_{z}=\mathrm{d}_{x} \hat{u}_{z}-\mathrm{d}_{x}\left(U \hat{b}_{z}\right)+\mathrm{d}_{x}\left(v_{\mathrm{m}} \mathrm{d}_{x} \hat{b}_{z}\right)+\mathrm{d}_{x}\left(\omega_{B e} \tau_{e e} v_{\mathrm{m}} \mathrm{d}_{x} \hat{b}_{y}\right), \\
& -\mathrm{i} \omega \hat{b}_{y}=\mathrm{d}_{x} \hat{u}_{y}-\mathrm{d}_{x}\left(U \hat{b}_{y}\right)+\mathrm{d}_{x}\left(v_{\mathrm{m}} \mathrm{d}_{y} \hat{b}_{y}\right)+\mathrm{d}_{x}\left(\omega_{B e} \tau_{e e} v_{\mathrm{m}} \mathrm{d}_{x} \hat{b}_{z}\right) .
\end{aligned}
$$

The generalization of the equations for the velocity oscillations Eq. (19) according the notations of Ref. [23] now reads

$$
\begin{aligned}
& \left(-\mathrm{i} \omega+U \mathrm{~d}_{x}\right) \hat{u}_{z}=V_{\mathrm{A}}^{2} \mathrm{~d}_{x} \hat{b}_{z}+\frac{1}{\rho} \mathrm{d}_{x}\left(\eta_{2} \mathrm{~d}_{x} \hat{u}_{z}\right)-\frac{1}{\rho} \mathrm{d}_{x}\left(\eta_{4} \mathrm{~d}_{x} \hat{u}_{y}\right), \\
& \left(-\mathrm{i} \omega+U \mathrm{~d}_{x}\right) \hat{u}_{y}=V_{\mathrm{A}}^{2} \mathrm{~d}_{x} \hat{b}_{y}+\frac{1}{\rho} \mathrm{d}_{x}\left(\eta_{2} \mathrm{~d}_{x} \hat{u}_{y}\right)+\frac{1}{\rho} \mathrm{d}_{x}\left(\eta_{4} \mathrm{~d}_{x} \hat{u}_{z}\right) .
\end{aligned}
$$

And the criterion to use approximation of viscous plasma with infinite conductivity becomes

$$
\varepsilon_{0} c^{2} \varrho_{\mathrm{H}}=\omega_{B e} \tau_{e e} \nu_{\mathrm{m}} \ll v_{\mathrm{k}}=\frac{\eta_{2}}{\rho},
$$

for $\omega_{B e} \tau_{e e}$. Substituting here for the Hall resistivity the $\varrho_{\mathrm{H}}=q_{e} n_{p} / B$ we obtain the criterion for infinite conductivity

$$
B \ll B_{\mathrm{H}}=H_{\mathrm{H}} / c \equiv \frac{q_{e} \eta_{2}(x)}{\varepsilon_{0} c^{2} M} .
$$

The magnetic field dependence of the reduced viscous coefficients $\eta_{2} / \eta$ and $\eta_{4} / \eta$ are

$$
\begin{aligned}
\frac{\eta_{2}}{\eta} & \approx \frac{\frac{6}{5} \tilde{x}^{2}+\frac{7}{3}}{\tilde{x}^{4}+4.03 \tilde{x}^{2}+\frac{7}{3}}, \\
\frac{\eta_{4}}{\eta} & \approx \frac{\left(\tilde{x}^{2}+\frac{7}{3}\right) \tilde{x}}{\tilde{x}^{4}+4.03 \tilde{x}^{2}+\frac{7}{3}} .
\end{aligned}
$$

If necessary, the frequency dependence of the kinetic coefficients can be also taken into account in the described system of equations describing the propagation of AW.

As the wind is along the magnetic field, the corresponding viscosity $\eta$ multiplying $\mathrm{d}_{x} U$ is not changed. The influence of the magnetic field on the kinetic coefficient creates coupling between the AW with different polarization and double the range of the matrix. Nevertheless the work with $8 \times 8$ matrices is a routine doable task after development of the system of notions and notations and qualitative analysis of the oversimplified examples. Qualitatively the dissipative kinetic coefficients $\eta$ and $\varrho$ transform the wave energy of waves propagating into an ideal fluid into wind energy of an ideal gas, and the contribution of the other components is negligible.

In short, the influence of the magnetic field on the profiles of temperature $T(x)$ and wind velocity $U(x)$ is reduced to decreasing of the slopes $\mathrm{d}_{x} T(x)$ and $\mathrm{d}_{x} U(x)$ beyond the TR in the beginning of the corona. 


\section{H Correlation energy}

For completely ionized plasma the correlation energy and pressure reads [58, 63, 64]

$$
\begin{aligned}
& E_{\text {corr }}=-e^{3} \sqrt{\frac{\pi}{T}} n^{3 / 2} V, \quad N=V n, \quad n=n_{e}+n_{p}, \quad n_{e}=n_{p}, \quad M=N\langle m\rangle, \\
& P=P_{\text {id }}+P_{\text {corr }}, \quad P_{\text {id }}=n T, \quad P_{\text {corr }}=-\frac{1}{3} \sqrt{\frac{\pi}{T}} e^{3} n^{3 / 2} .
\end{aligned}
$$

Then for the correlation part of the enthalpy per unit mass we have

$$
h=\frac{E+P V}{n V\langle m\rangle}=h_{\mathrm{id}}+h_{\mathrm{corr}}, \quad h_{\mathrm{id}}=c_{p} \frac{T}{\langle m\rangle}, \quad h_{\mathrm{corr}}=-\frac{4 \sqrt{\pi}}{3} \frac{e^{3}}{\langle m\rangle} \sqrt{\frac{n}{T}},
$$

and for the dimensionless energy and momentum fluxes we obtain

$$
\begin{aligned}
\chi_{\mathrm{corr}}(x) & =\frac{U(0) h_{\mathrm{corr}}(0)-U(x) h_{\mathrm{corr}}(x)}{\rho_{0} U_{0}^{3}}, \\
\tau_{\mathrm{corr}}(x) & =\frac{p_{\text {corr }}(0)-p_{\text {corr }}(x)}{\rho_{0} U_{0}^{2}} .
\end{aligned}
$$

In such a way the Debye-Hückel correlation effects in solar plasma are incorporated in the developed scheme. To every term of the momentum flux we can ascribe corresponding volume density of a force $f_{i}=\partial_{k} \sigma_{k i}[65]$ and the correlation correction of the pressure is not an exception $f_{\text {corr }}=-\nabla p_{\text {corr }}$. 